\title{
The normal chemistry of multiple stellar populations in the dense globular cluster NGC 6093 (M 80) ${ }^{\star, \star \star, \star \star \star}$
}

\author{
E. Carretta ${ }^{1}$, A. Bragaglia ${ }^{1}$, R. G. Gratton ${ }^{2}$, V. D’Orazi ${ }^{2,3,4}$, S. Lucatello ${ }^{2}$, A. Sollima ${ }^{1}$, Y. Momany ${ }^{2,5}$, \\ G. Catanzaro ${ }^{6}$, and F. Leone ${ }^{7}$
}

1 INAF-Osservatorio Astronomico di Bologna, via Ranzani 1, 40127 Bologna, Italy e-mail: eugenio.carretta@oabo.inaf.it

2 INAF-Osservatorio Astronomico di Padova, Vicolo dell'Osservatorio 5, 35122 Padova, Italy

3 Dept. of Physics and Astronomy, Macquarie University, NSW 2109 Sydney, Australia

4 Monash Centre for Astrophysics, Monash University, School of Mathematical Sciences, Building 28, Clayton VIC 3800 , Melbourne, Australia

5 European Southern Observatory, Alonso de Cordova 3107, Vitacura, Santiago, Chile

6 INAF-Osservatorio Astrofisico di Catania, via S.Sofia 78, 95123 Catania, Italy

7 Dipartimento di Fisica e Astronomia, Università di Catania, via S.Sofia 78, 95123 Catania, Italy

Received 24 February 2015 / Accepted 6 March 2015

\begin{abstract}
We present the abundance analysis of 82 red giant branch stars in the dense, metal-poor globular cluster NGC 6093 (M 80), the largest sample of stars analysed in this way for this cluster. From high-resolution UVES spectra of 14 stars and intermediate resolution GIRAFFE spectra for the other stars we derived abundances of $\mathrm{O}, \mathrm{Na}, \mathrm{Mg}, \mathrm{Al}, \mathrm{Si}, \mathrm{Ca}, \mathrm{Sc}, \mathrm{Ti}, \mathrm{V}, \mathrm{Cr}, \mathrm{Mn}, \mathrm{Fe}, \mathrm{Co}, \mathrm{Ni}, \mathrm{Cu}, \mathrm{Zn}, \mathrm{Y}, \mathrm{Zr}$, $\mathrm{Ba}, \mathrm{La}, \mathrm{Ce}, \mathrm{Pr}, \mathrm{Nd}, \mathrm{Sm}$, Eu. On our UVES metallicity scale the mean metal abundance of $\mathrm{M} 80$ is $[\mathrm{Fe} / \mathrm{H}]=-1.791 \pm 0.006 \pm 0.076$ ( \pm statistical \pm systematic error) with $\sigma=0.023$ (14 stars). M 80 shows star-to-star variations in proton-capture elements, and the extension of the Na-O anti-correlation perfectly fit the relations with (i) total cluster mass; (ii) horizontal branch morphology; and (iii) cluster concentration previously found by our group. The chemistry of multiple stellar populations in M 80 does not look extreme. The cluster is also a representative of halo globular clusters concerning the pattern of $\alpha$-capture and Fe-group elements. However we found that a significant contribution from the $s$-process is required to account for the distribution of neutron-capture elements. A minority of stars in M 80 seem to exhibit slightly enhanced abundances of $s$-process species, compatible with those observed in M 22 and NGC 1851, although further confirmation from larger samples is required.
\end{abstract}

Key words. stars: abundances - stars: Population II - globular clusters: general - globular clusters: individual: NGC 6093 (M 80) stars: atmospheres

\section{Introduction}

The observational evidence of multiple stellar populations in Galactic globular clusters (GCs) was already available since the late '70s, in the large star-to-star abundance variations of light elements $(\mathrm{C}, \mathrm{N}, \mathrm{O}, \mathrm{Na}, \mathrm{Mg}, \mathrm{Al})$ following patterns of correlations and anti-correlations (see reviews by Smith 1987; Kraft 1994; Sneden 2000; Gratton et al. 2004). However, Denisenkov \& Denisenkova (1989) and Langer et al. (1993) did not explain the observed abundance variations, with the simultaneous action of the $\mathrm{Ne}-\mathrm{Na}$ and $\mathrm{Mg}-\mathrm{Al}$ chains in the same temperature layers where the ON part of the complete CNO cycle was at work, until the late 80s and early 90s. The discovery of $\mathrm{Na}-\mathrm{O}$ and $\mathrm{Mg}-\mathrm{Al}$

\footnotetext{
* Based on observations collected at ESO telescopes under programme 083.D-0208.

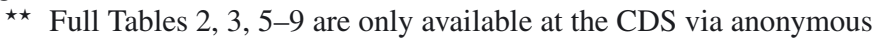
ftp to cdsarc.u-strasbg. fr (130.79.128.5) or via

http://cdsarc.u-strasbg.fr/viz-bin/qcat?]/A+A/578/A116

$\star \star \star$ Appendix A is available in electronic form at

http://www . aanda.org
}

anti-correlation among turn-off and subgiant branch (SGB) stars first in NGC 6752 (Gratton et al. 2001) and then in other GCs (M 71, Ramírez \& Cohen 2002; M 5, Ramírez \& Cohen 2003; 47 Tuc, Carretta et al. 2004) provided the key spectroscopic evidence of multiple populations. These unevolved stars can neither synthesise nor transport these elements to the surface, thus they must be formed from gas polluted by ejecta of more massive stars of an earlier stellar generation. Hence, "observing an anti-correlation among proton-capture elements" is simply a rewording for "seeing multiple stellar populations".

In the last ten years, the advent of efficient, highmultiplexing spectrographs permitted astronomers to collect precise abundances for large samples of stars from high resolution spectroscopy. We exploited FLAMES at VLT and designed a large, systematic survey of red giant branch (RGB) stars in Galactic GCs (for results, see e.g. Carretta et al. 2006, 2009a,b,c, 2014a,b,c; and references therein; D'Orazi et al. 2010a, 2014; Gratton et al. 2006, 2007). This investigation was recently extended to other evolutionary phases like horizontal branch (HB), SGB, and dwarf stars (e.g. D'Orazi et al. 2010b; Gratton et al. 2011, 2012a, 2014). Other, independent studies concentrated 
mostly on the closest GCs like 47 Tuc (e.g. Cordero et al. 2014; Dobrovolskas et al. 2014), M 4 (e.g. D’Orazi \& Marino 2010; D'Orazi et al. 2013; Marino et al. 2008), NGC 6397 (e.g. Lind et al. 2008, 2009; González Hernández et al. 2009; Lovisi et al. 2012), NGC 6752 (e.g. Yong et al. 2005, 2013; Gruyters et al. 2014; Shen et al. 2010), and M 22 (Marino et al. 2011, 2012, 2013). The literature is vast ${ }^{1}$ and the reader is directed to the review by Gratton et al. (2012b) and to the references in the more recent papers.

Our homogenous analysis of a large sample of GCs and large numer of stars in each GC permits a quantitative approach (e.g. Carretta et al. 2009a,b), showing that the Na-O anti-correlation differs from cluster to cluster in shape and extent (measured by the interquartile range $-\mathrm{IQR}$ - of the $[\mathrm{O} / \mathrm{Na}]$ ratio, introduced by Carretta 2006). Adding also literature data, we see that the $\mathrm{Na}-\mathrm{O}$ anti-correlation stands out as the chief chemical signature of multiple stellar populations, so widespread that it may even be used to define a GC and that the phenomenon is primarily driven by the cluster mass (Carretta et al. 2010a). However, our ignorance about the still elusive nature of first generation polluters hampers our vision. Massive stars, either single, and fast rotating (FRMS: Decressin et al. 2007) or in close binary systems (de Mink et al. 2009), intermediate-mass asymptotic giant branch (IM-AGB) and super-AGB stars (Ventura et al. 2001; D'Ercole et al. 2012), or none of them (Bastian et al. 2013), have been proposed as polluters. It is even possible that both the two commonly favoured candidates (FRMS and IM-AGB) were at work. In two GCs where RGB stars were recently found clustered into discrete groups according to their distinct composition (NGC 6752: Carretta et al. 2012; NGC 2808: Carretta 2014) the population with intermediate chemistry cannot be reproduced by mixing unprocessed and heavily polluted matter from the primordial and the extremely processed components, calling for at least two kind of polluters.

Our approach to these problems has been to gather an unprecedented large statistics, both in term of number of GCs and of stars for which homogeneous abundances were derived in each GC. We studied 24 massive GCs with different global parameters (metallicity, age, Galactic population, HB morphology, concentration, etc.) and we present here the abundance analysis of the last of them, NGC 6093 (M 80).

M 80 is a metal-poor $([\mathrm{Fe} / \mathrm{H}]=-1.73$ dex, Cavallo et al. 2004), moderately massive $\left(M_{V}=-8.23 \mathrm{mag}\right.$, Harris 1996 , 2010 edition) cluster of Oosterhoff type II (Kopacki 2013), one of the 30 densest GCs in the Milky Way. In the central regions of M 80 Ferraro et al. (1999) identified over 300 blue straggler stars, despite the relatively low inferred collision rate. Their inference was that $M 80$ could be in an unusual dynamical state, on the edge of core collapse where the density has recently become large enough to increase the encounters involving primordial binaries, triggering a burst of blue straggler formation (but see Heinke et al. 2003 for a different view, based on the comparison with 47 Tuc and its possibly uncomplete census of blue stragglers). The HB morphology is characterised by an extended blue

\footnotetext{
1 We concentrate on spectroscopy in the present paper. However, improvements in precision photometry from ground- or space-based facilities produced colour-magnitude diagrams (CMDs) of GCs revealing more and more split SGBs, RGBs, and even multiple main sequences (e.g. Grundahl et al. 1998; Han et al. 2009; Lee et al. 2009; Monelli et al. 2013; Milone et al. 2012, 2013; Nardiello et al. 2015 and references therein). The synergy between spectroscopy and photometry allows the uncovering of how absorption features due to the involved light elements affect the colour indexes of split sequences (e.g. Carretta et al. 2011a; Sbordone et al. 2011; Milone et al. 2012; Cassisi et al. 2013).
}

Table 1. Log of FLAMES observations for NGC 6093.

\begin{tabular}{lccccc}
\hline \hline $\begin{array}{l}\text { Date } \\
\text { (yyyy-mm-dd) }\end{array}$ & $\begin{array}{c}\text { UT } \\
\text { (hh:mm:ss) }\end{array}$ & $\begin{array}{c}\text { Exp. } \\
\text { (s) }\end{array}$ & Setup & $\begin{array}{c}\text { Seeing } \\
\left({ }^{\prime \prime}\right)\end{array}$ & Airmass \\
\hline $2009-09-08$ & $00: 28: 42.650$ & 2940 & HR11 & 0.89 & 1.237 \\
$2009-09-09$ & $00: 50: 31.604$ & 2940 & HR11 & 0.91 & 1.343 \\
$2009-08-06$ & $00: 16: 09.457$ & 2940 & HR11 & 0.95 & 1.002 \\
$2009-09-10$ & $00: 02: 22.285$ & 2940 & HR13 & 1.44 & 1.177 \\
$2009-09-12$ & $23: 40: 18.289$ & 2940 & HR13 & 0.86 & 1.149 \\
$2009-08-06$ & $00: 16: 09.457$ & 2940 & HR13 & 0.94 & 1.330 \\
\hline
\end{tabular}

tail (Ferraro et al. 1998), with the concentration of blue tail stars increasing toward the cluster centre (Brocato et al. 1998). M 80 presents a typical halo-type orbit, with a short period and small size, never leaving the inner 3-4 kpc of the Galaxy (Dinescu et al. 1999).

The only chemical evidence of multiple populations comes from the study of ten RGB stars by Cavallo et al. (2004) who found a mean $[\mathrm{Al} / \mathrm{Fe}]$ ratio of +0.37 dex with a large spread of 0.43 dex (1 sigma) in aluminum abundance. Recently Monelli et al. (2013) used private photometry from their SUMO project to study the colour spread of the RGB.

The paper is organised as follows: in Sect. 2 we present the basic data for this cluster, the selection of stars to be observed, and the observations themselves. In Sect. 3 we describe the analysis method, and the results for abundances are presented in Sect. 4. The results are discussed in Sect. 5 in the context of multiple populations in GCs. Finally, in Sect. 6 we summarise our findings.

\section{Observations}

The photometric catalog for NGC 6093 is based on $B V$ data collected at the Wide-Field Imager at the 2.2-m ESO-MPI telescope on 9 July 1999. These data were reduced by YM using a standard method (see Carretta et al. 2014a; Momany et al. 2004 for details) and the photometry is unpublished.

Following our standard procedure, we selected a pool of stars lying near the RGB ridge line in the CMD and without close neighbours. The stars in our spectroscopic sample are indicated as large symbols in Fig. 1a, with a zooming of the RGB region in Fig. 1b, where we also indicate the membership status of our targets (based on the analysis of their spectra). NGC 6093 is heavily contaminated by field stars. The excision of non-members was generally done via radial velocity (RV); however, in some cases, we discarded stars on the basis of their metallicity (see next sections).

We used FLAMES at VLT and the log of the observations is given in Table 1. We obtained three exposures with the highresolution GIRAFFE grating and the setup HR11 covering the Na I 5682-88 $\AA$ doublet and three exposures with the setup HR13 including the [O I] forbidden lines at 6300-63 $\AA$ and another Na I doublet at 6154-60 $\AA$. We observed a total of 14 giants with the fibres feeding the UVES spectrograph (Red Arm, with spectral range from 4800 to $6800 \AA$ and $R=47000)$. All 14 stars turned out to be members. We observed 78 member stars of M 80 with GIRAFFE; ten are in common with UVES, so the grand total of our sample consists of 82 red giant stars.

We used the 1D, wavelength calibrated spectra as reduced by the ESO personnel with the dedicated FLAMES pipelines. The 

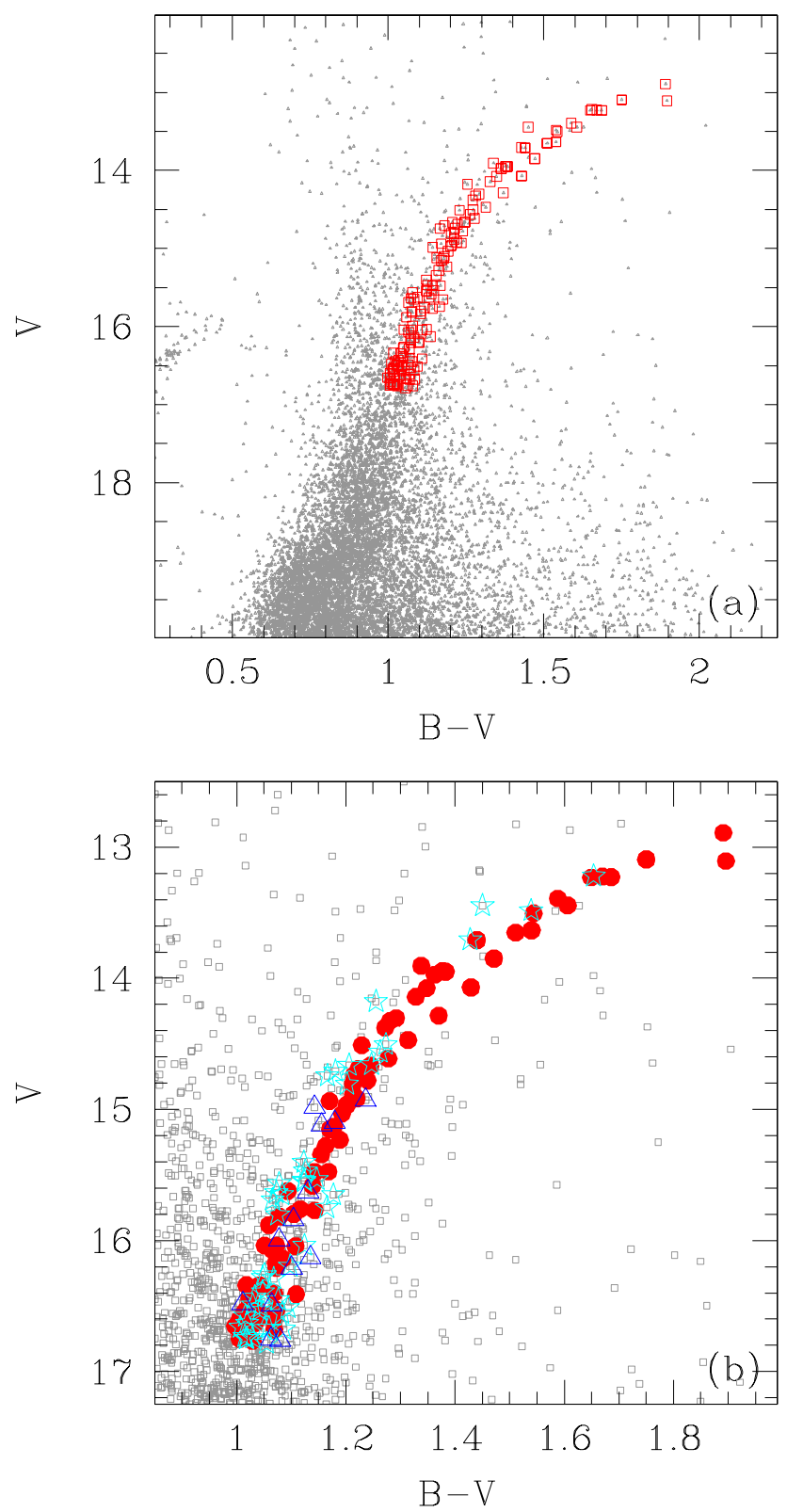

Fig. 1. a) CMD of the cluster, with FLAMES targets indicated by red, open symbols. b) Enlargement of the RGB region, with member stars indicated with filled red dots, non-members on the basis of RV with cyan open stars, non-members on the basis of abundance with open blue triangles.

RVs for stars observed with the GIRAFFE spectrograph were obtained using the IRAF ${ }^{2}$ task FXCORR, with appropriate templates, while those of the stars observed with UVES were derived with the IRAF task RVIDLINES. The multiple exposures were then combined. The median values of the signal-to-noise ratio of the combined spectra are 125,68 , and 78 , for observations with UVES and with the GIRAFFE HR11 and HR13 setups, respectively.

Our optical $B, V$ photometric data were integrated with $K$-band magnitudes from the Point Source Catalog of 2MASS

\footnotetext{
2 IRAF is distributed by the National Optical Astronomical Observatory, which are operated by the Association of Universities for Research in Astronomy, under contract with the National Science Foundation.
}
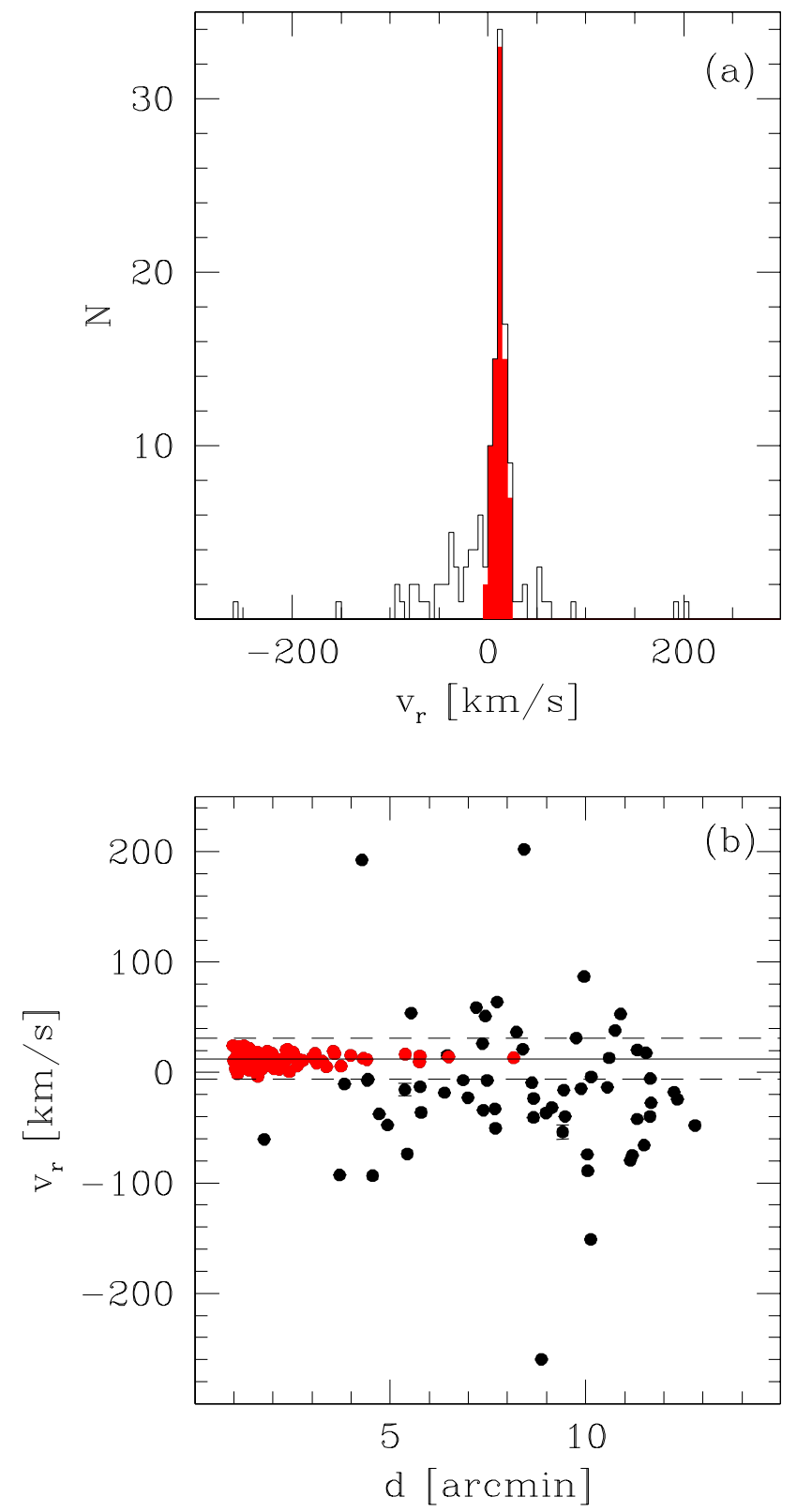

Fig. 2. a) Histogram of RV values for all stars observed. b) Plot of RV as function of distance from the cluster centre; we indicate the member stars in red. The lines show the average RV and the $\pm 3 \sigma$ limits.

(Skrutskie et al. 2006) to derive atmospheric parameters as described in Sect. 3.

Coordinates, magnitudes, and heliocentric RVs are shown in Table 2.

According to Harris (1996, 2010 edition), the metallicity of NGC 6093 is -1.75 and the RV is $8.1 \pm 1.5 \mathrm{~km} \mathrm{~s}^{-1}$. Our choice of member stars rests both on RV and, for marginal or dubious cases, also on metallicity. Figure 2 shows the histogram of the heliocentric RVs (in panel a) and the run of RVs with distance from the cluster centre (panel b). We selected 82 stars for which both RV and metallicity were acceptable (see Table 2) as candidate cluster members. Not surprisingly, they are concentrated at small distances from the cluster centre. For those candidates, we find an average velocity of $\langle\mathrm{RV}\rangle=11.9 \pm 0.7 \mathrm{~km} \mathrm{~s}^{-1}$ $\left(\mathrm{rms}=6.1 \mathrm{~km} \mathrm{~s}^{-1}\right)$, in good agreement with the value reported by Harris (1996). 
Table 2. List and relevant information for target stars in NGC 6093.

\begin{tabular}{lccccccrrr}
\hline \hline ID & $\begin{array}{c}\text { RA } \\
\text { HH:MM:SS }\end{array}$ & $\begin{array}{c}\text { Dec } \\
\text { DD:PP:SS }\end{array}$ & $B$ & $V$ & Setup & $\begin{array}{r}\mathrm{RV}_{H} \\
\mathrm{~km} \mathrm{~s}^{-1}\end{array}$ & $\begin{array}{r}\text { Err(RV) } \\
\mathrm{km} \mathrm{s}^{-1}\end{array}$ & $\begin{array}{c}\text { Dist } \\
\operatorname{arcmin}\end{array}$ \\
\hline \multicolumn{7}{c}{ Members on the basis of radial velocity and metallicity } \\
2174 & 161658.972 & -225315.440 & 17.113 & 16.040 & HR11,13 & 16.45 & 0.18 & 5.37 \\
2232 & 161704.518 & -225250.850 & 16.877 & 15.760 & HR11,13 & 14.96 & 0.03 & 5.74 \\
10043 & 161642.766 & -230209.290 & 16.643 & 15.475 & HR13 & 9.74 & 0.60 & 5.77 \\
11607 & 161719.568 & -230543.100 & 16.914 & 15.771 & HR11,13 & 13.26 & 0.47 & 8.17 \\
11776 & 161652.809 & -230441.900 & 15.606 & 14.325 & HR11,13 & 14.39 & 0.13 & 6.52 \\
\hline
\end{tabular}

Notes. The complete table is available at CDS.

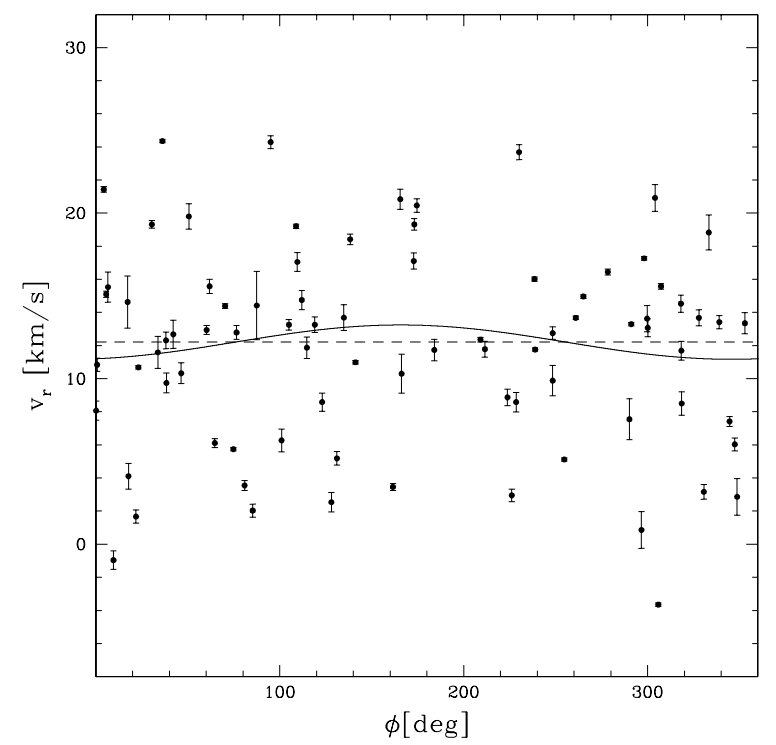

Fig. 3. Radial velocities of member stars in M 80 as a function of their position angle.

\subsection{Kinematics}

The spectroscopic data set presented here constitutes the largest sample of intermediate and high resolution spectra collected so far for NGC 6093, and can therefore be used to study the kinematical properties of this cluster.

In Fig. 3 the radial velocities of the member stars are plotted against their position angle. To test the possible presence of systemic rotation, we calculated the difference between the average velocity of stars located within and outside a $180^{\circ}$ interval centred around various position angles and compared it with a set of Monte Carlo extractions of the same number of stars randomly distributed across the field of view. We found no significant signature of rotation with a maximum difference lying at $\sim 1 \sigma$ from the mean distribution of randomly extracted stars. The best-fit sinusoidal curve overplotted to Fig. 3 has a maximum amplitude of $A_{\text {rot }}=1.0 \pm 0.6 \mathrm{~km} \mathrm{~s}^{-1}$, corresponding to a ratio $A_{\text {rot }} / \sigma_{0}=0.09 \pm 0.06$ (see below). This value places NGC 6093 in the group of non-rotating or slowly rotating GCs of Bellazzini et al. (2012).

We then fitted the radial distribution of velocities with a set of single-mass King (1966) and multi-mass King-Michie (Gunn \& Griffin 1979) isotropic models. For this purpose, the density profile has been derived by counting stars brighter than the turnoff point (well above the limiting magnitude of the WFI photometry where severe incompleteness affects star counts) in concentric annuli at different distance to the cluster centre.
We then matched the obtained density profile with that provided by Noyola \& Gebhardt (2006) from integrated photometry on Hubble Space Telescope images to sample the innermost 1.7' where the WFI spatial resolution does not allow a complete sampling of stars. The total cluster $V$ magnitude, obtained by integrating the observed profile, turns out to be $V=7.48 \pm 0.05$, corresponding to a total luminosity of $\log L / L_{\odot}=5.18 \pm 0.09$. We derived the velocity dispersion profile by dividing the sample in four radial bins containing $\sim 20$ stars each.

We constructed multi-mass models assuming nine mass bins and a mass function derived iteratively to match the observed star counts in the deep ACS photometry of NGC 6093 from the ACS globular clusters treasury project (Sarajedini et al. 2007) corrected for completeness (see Anderson et al. 2008). We adopted the same procedure described in Sollima et al. (2012) to account for dark remnants and binary heating. We calculated the dynamical masses of the best-fit models using the maximum likelihood algorithm described by Pryor \& Meylan (1993) applied to our sample of radial velocities. The estimated central line of sight (LOS) velocity dispersions and the corresponding dynamical masses are $\sigma_{v, 0}=11.4 \pm 0.9 \mathrm{~km} \mathrm{~s}^{-1}$ and $\log M / M_{\odot}=$ $5.50 \pm 0.07$ for the single-mass model and $\sigma_{v, 0}=10.1 \pm 0.8 \mathrm{~km} \mathrm{~s}^{-1}$ $\log M / M_{\odot}=5.56 \pm 0.07$ for the multi-mass model. The bestfit models are overplotted to the density and velocity dispersion profiles in Fig. 4. It is apparent that both models provide a good fit of both profiles. The resulting $\mathrm{M} / \mathrm{L}$ ratio is $2.1 \pm 0.6$, slightly larger than that predicted for a mass-follow-light stellar system with the mass function slope estimated in the literature ( $\alpha=-1.36$; Paust et al. 2010), although the large associated error prevents any firm conclusion. This is in agreement with the findings of Sollima et al. (2012) for a sample of six Galactic GCs, which are interpreted as a consequence of a high retention efficency of dark remnants and/or to tidal heating. In this regard, NGC 6093 follows an eccentric orbit $(e=0.85)$ in an inner region of the Milky Way (Dinescu et al. 1999) being therefore subject to an intense tidal stress that can inflate its velocity dispersion even within its half mass (Küpper et al. 2010).

\section{Atmospheric parameters, metallicity, and abundance analysis}

In the abundance analysis, we used mostly equivalent widths (EWs) that are measured as described in detail in Bragaglia et al. (2001) using the package ROSA (Gratton 1988). The EWs measured on the GIRAFFE spectra were shifted to a system defined by EWs from the high-resolution UVES spectra. For this correction, we used 275 lines measured in the 10 stars observed with both instruments.

Abundances were derived from EW analysis, using the Kurucz (1993) grid of solar-scaled local thermodynamical 

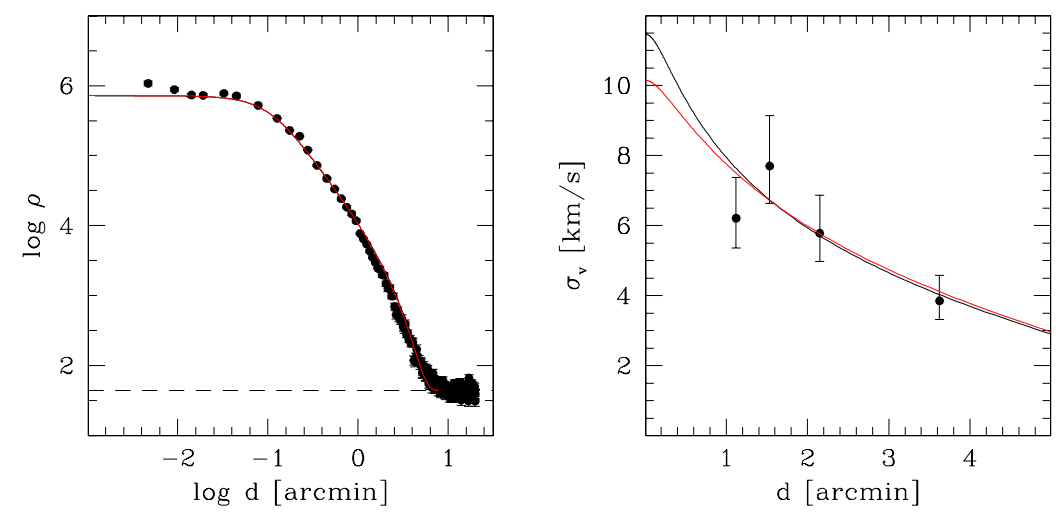

Fig. 4. Density and velocity dispersion profiles (left and right panel, respectively) in M 80. The best-fit models are superimposed (solid red line).

Table 3. Adopted atmospheric parameters and derived iron abundances in NGC 6093.

\begin{tabular}{lcccccccccc}
\hline \hline Star & $\begin{array}{c}T_{\text {eff }} \\
(\mathrm{K})\end{array}$ & $\begin{array}{c}\log g \\
(\mathrm{dex})\end{array}$ & $\begin{array}{c}{[\mathrm{A} / \mathrm{H}]} \\
(\mathrm{dex})\end{array}$ & $\begin{array}{c}v_{\mathrm{t}} \\
\left(\mathrm{km} \mathrm{s}^{-1}\right)\end{array}$ & $\begin{array}{c}{[\mathrm{Fe} / \mathrm{H}] \mathrm{I}} \\
(\mathrm{dex})\end{array}$ & $\mathrm{rms}$ & $n$ & $\begin{array}{r}{[\mathrm{Fe} / \mathrm{HII}} \\
(\mathrm{dex})\end{array}$ & $\mathrm{rms}$ \\
\hline 2174 & 4973 & 2.30 & -1.73 & 1.70 & 21 & -1.729 & 0.118 & 2 & -1.697 & 0.073 \\
2232 & 4887 & 2.12 & -1.76 & 1.84 & 22 & -1.757 & 0.068 & 1 & -1.773 & \\
10043 & 4829 & 2.00 & -1.78 & 2.11 & 17 & -1.776 & 0.077 & 3 & -1.787 & 0.059 \\
11607 & 4905 & 2.17 & -1.77 & 1.82 & 22 & -1.773 & 0.085 & 1 & -1.795 & \\
11776 & 4546 & 1.46 & -1.81 & 0.88 & 37 & -1.807 & 0.155 & 2 & -1.810 & 0.031 \\
\hline
\end{tabular}

Notes. The parameter $n$ is the number of lines used in the analysis. The complete table is available at CDS.

equilibrium (LTE) model atmospheres with the overshooting option switched off.

We derived effective temperatures $T_{\text {eff }}$ following our usual two-step procedure: first pass values were obtained from $V-K$ colours and the Alonso et al. $(1999,2001)$ calibration, and these values were used as inputs to derive the finally adopted $T_{\text {eff' }}$ 's from a relation between $T_{\text {eff }}(V-K)$ and the star magnitudes. Given the non-negligible value of the reddening for M 80 $(E(B-V)=0.18 \mathrm{mag}$, Harris 1996), we employed near-infrared $K$ magnitudes for this relation.

Surface gravities $\log g$ were obtained from bolometric corrections (from Alonso et al.), the adopted effective temperatures, reddening, and distance modulus from Harris (1996), and assuming masses of $0.85 M_{\odot}$ and $M_{\mathrm{bol}, \odot}=4.75$ as bolometric magnitude for the Sun.

Finally, we obtained values of the microturbulent velocity $v_{\mathrm{t}}$ by eliminating trends of the abundances from $\mathrm{Fe}$ I lines with the expected line strength (see Magain 1984). The final adopted atmospheric parameters are listed with the derived $\mathrm{Fe}$ abundances of individual stars in Table 3.

Errors in atmospheric parameters and their impact on the derived abundances are estimated as usual (see Carretta et al. 2009a,b and the Appendix to the present paper). Internal (star to star) errors in temperature, gravity, model abundance, and $v_{t}$ are $4 \mathrm{~K}, 0.04 \mathrm{dex}, 0.02 \mathrm{dex}$, and $0.10 \mathrm{~km} \mathrm{~s}^{-1}$, respectively ${ }^{3}$.

As in the vast majority of GCs, the metallicity is very homogeneous in $\mathrm{M} 80$. We find on average $[\mathrm{Fe} / \mathrm{H}] \mathrm{I}=-1.791 \pm 0.006 \pm$ $0.076 \operatorname{dex}(\sigma=0.023$ dex $)$ from the 14 stars observed with UVES and $[\mathrm{Fe} / \mathrm{H}] \mathrm{I}=-1.791 \pm 0.003 \pm 0.070 \mathrm{dex}(\sigma=0.023 \mathrm{dex})$ from 78 stars with GIRAFFE spectra (where the first and second error bars refer to the statistical and systematic errors, respectively). The agreement with average abundances derived from singly ionised $\mathrm{Fe}$ lines is very good: $[\mathrm{Fe} / \mathrm{H}] \mathrm{II}=-1.791 \mathrm{dex}$

\footnotetext{
3 The internal error in $v_{\mathrm{t}}$ increases to $0.36 \mathrm{~km} \mathrm{~s}^{-1}$ for stars with GIRAFFE spectra.
}

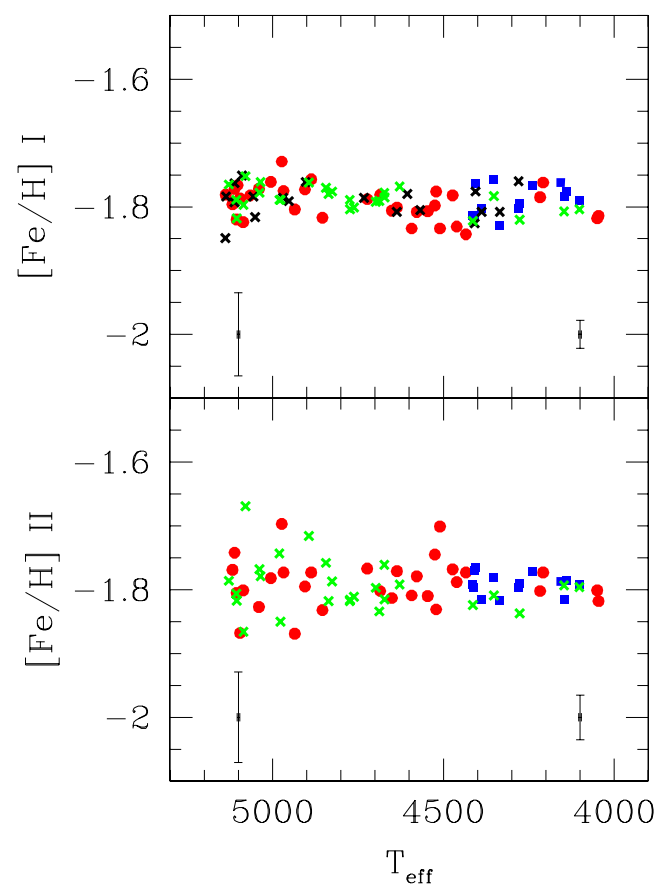

Fig. 5. Abundance ratios $[\mathrm{Fe} / \mathrm{H}]$ I (upper panel) and $[\mathrm{Fe} / \mathrm{H}]$ II (lower panel) as a function of $T_{\text {eff }}$ for all member stars analysed in $\mathrm{M} 80$ with UVES (blue squares) and GIRAFFE spectra (red circles: setups HR11 and HR13; black crosses: HR11 only; green crosses: HR13 only). Error bars on the right and left side are star-to-star errors for targets observed with UVES and GIRAFFE, respectively.

$(\sigma=0.016$ dex, 14 stars with UVES $)$ and $[\mathrm{Fe} / \mathrm{H}] \mathrm{II}=-1.792 \mathrm{dex}$ ( $\sigma=0.040$ dex, 56 stars with GIRAFFE). The derived abundances show no trend as a function of the temperature (Fig. 5) and are in good agreement, within the uncertainties, with the average value found by Cavallo et al. (2004) from ten giants. 
Table 4. Mean abundances from UVES and GIRAFFE.

\begin{tabular}{|c|c|c|}
\hline \multirow[t]{2}{*}{ Element } & UVES & GIRAFFE \\
\hline & $n$ avg rms & $n$ avg rms \\
\hline$[\mathrm{O} / \mathrm{Fe}] \mathrm{I}$ & $14+0.240 .24$ & $54+0.230 .21$ \\
\hline$[\mathrm{Na} / \mathrm{Fe}] \mathrm{I}$ & $14+0.440 .22$ & $63+0.400 .30$ \\
\hline$[\mathrm{Mg} / \mathrm{Fe}] \mathrm{I}$ & $14+0.450 .10$ & $66+0.460 .05$ \\
\hline$[\mathrm{Al} / \mathrm{Fe}] \mathrm{I}$ & $14+0.480 .34$ & \\
\hline$[\mathrm{Si} / \mathrm{Fe}] \mathrm{I}$ & $14+0.340 .04$ & $75+0.360 .03$ \\
\hline$[\mathrm{Ca} / \mathrm{Fe}] \mathrm{I}$ & $14+0.360 .02$ & $78+0.360 .02$ \\
\hline$[\mathrm{Sc} / \mathrm{Fe}] \mathrm{II}$ & $14-0.030 .03$ & $78-0.010 .03$ \\
\hline$[\mathrm{Ti} / \mathrm{Fe}] \mathrm{I}$ & $14+0.190 .05$ & $64+0.190 .02$ \\
\hline$[\mathrm{Ti} / \mathrm{Fe}] \mathrm{II}$ & $14+0.180 .02$ & \\
\hline$[\mathrm{V} / \mathrm{Fe}] \mathrm{I}$ & $14-0.040 .02$ & $45-0.040 .03$ \\
\hline$[\mathrm{Cr} / \mathrm{Fe}] \mathrm{I}$ & $14-0.050 .03$ & $34-0.030 .04$ \\
\hline$[\mathrm{Cr} / \mathrm{Fe}] \mathrm{II}$ & $14-0.010 .03$ & \\
\hline$[\mathrm{Mn} / \mathrm{Fe}] \mathrm{I}$ & $14-0.480 .02$ & \\
\hline$[\mathrm{Fe} / \mathrm{H}] \mathrm{I}$ & $14-1.790 .02$ & $78-1.790 .02$ \\
\hline$[\mathrm{Fe} / \mathrm{H}] \mathrm{II}$ & $14-1.790 .02$ & $56-1.790 .04$ \\
\hline$[\mathrm{Co} / \mathrm{Fe}] \mathrm{I}$ & $14-0.220 .03$ & \\
\hline$[\mathrm{Ni} / \mathrm{Fe}] \mathrm{I}$ & $14-0.130 .02$ & $69-0.130 .02$ \\
\hline$[\mathrm{Cu} / \mathrm{Fe}] \mathrm{I}$ & $14-0.520 .04$ & \\
\hline$[\mathrm{Zn} / \mathrm{Fe}] \mathrm{I}$ & $14-0.020 .06$ & \\
\hline [Y/Fe]II & $14-0.070 .04$ & \\
\hline$[\mathrm{Zr} / \mathrm{Fe}] \mathrm{I}$ & $8-0.030 .05$ & \\
\hline$[\mathrm{Zr} / \mathrm{Fe}] \mathrm{II}$ & $14+0.000 .06$ & \\
\hline$[\mathrm{Ba} / \mathrm{Fe}] \mathrm{II}$ & $14+0.160 .16$ & $59+0.120 .28$ \\
\hline [La/Fe]II & $14+0.280 .11$ & \\
\hline$[\mathrm{Ce} / \mathrm{Fe}] \mathrm{II}$ & $14+0.180 .14$ & \\
\hline$[\mathrm{Pr} / \mathrm{Fe}] \mathrm{II}$ & $14+0.240 .09$ & \\
\hline$[\mathrm{Nd} / \mathrm{Fe}] \mathrm{II}$ & $14+0.220 .08$ & \\
\hline$[\mathrm{Sm} / \mathrm{Fe}] \mathrm{II}$ & $14+0.400 .08$ & \\
\hline$[\mathrm{Eu} / \mathrm{Fe}] \mathrm{II}$ & $14+0.510 .02$ & \\
\hline
\end{tabular}

We measured the abundances of 12 species (beside Fe: O, $\mathrm{Na}, \mathrm{Mg}, \mathrm{Si}, \mathrm{Ca}, \mathrm{Ti}, \mathrm{Sc}, \mathrm{V}, \mathrm{Cr}, \mathrm{Ni}$, and $\mathrm{Ba}$ ) from both UVES and GIRAFFE spectra. Additionally, from the UVES spectra with large spectral coverage, we also obtained abundances of $\mathrm{Al}$, Ti (from both $\mathrm{Ti}$ I and Ti II lines), $\mathrm{Cr}$ (both from $\mathrm{Cr}$ I and Cr II lines), Mn, Co, Cu, $\mathrm{Zn}, \mathrm{Zr}$ (both from $\mathrm{Zr}$ I and $\mathrm{Zr}$ II lines), $\mathrm{La}, \mathrm{Ce}, \mathrm{Pr}, \mathrm{Nd}, \mathrm{Sm}$, and $\mathrm{Eu}$. Adopted line list and solar reference abundances are from Gratton et al. (2003). We applied non-LTE corrections to the $\mathrm{Na}$ abundances following the prescriptions by Gratton et al. (1999). We applied abundance corrections for Sc, $\mathrm{V}, \mathrm{Mn}$, and Co to account for the hyperfine structure (references in Gratton et al. 2003).

\section{Results}

The average values of all measured elements with their rms scatter are listed in Table 4. Abundances of proton-capture, $\alpha$-capture, Fe-group, and neutron-capture elements are given for individual stars in Tables 5-9. Abundance ratios as a function of temperature are shown in Fig. 6. In this figure, we also plot the average $[\alpha / \mathrm{Fe}]$ values, where the mean includes $[\mathrm{Si} / \mathrm{Fe}],[\mathrm{Ca} / \mathrm{Fe}]$, and $[\mathrm{Ti} / \mathrm{Fe}] \mathrm{I}$.

\subsection{Proton-capture elements}

The Na I lines at 6154-60 $\AA$ are weak in metal-poor, relatively warm stars, as discussed in Carretta et al. (2009a). The possibility that they are measured only when noise spuriously enhances their strength prompted us to adopt an empirical parameter $\left(T_{\text {eff }} / 100 \mathrm{~K}-10 \times[\mathrm{Fe} / \mathrm{H}]\right)$ to set rejection criteria in these cases. We found that 12 stars in M 80 (mostly warmer than $\sim 4800 \mathrm{~K}$ and with $[\mathrm{Na} / \mathrm{Fe}] \geq 0.5 \mathrm{dex}$, with only one or both $\mathrm{Na}$ lines from the weak doublet, had the parameter exceeding the value of 65 fixed in Carretta et al. (2009a). For sake of homogeneity, we dropped the $\mathrm{Na}$ abundance in these stars, although all these objects have a measured $\mathrm{O}$ abundance that would put them reasonably well on the Na-O anti-correlation. After this culling of our sample, we ended with $\mathrm{O}$ abundances for 63 RGB stars (44 actual detections and 19 upper limits) and $\mathrm{Na}$ abundances for 67 stars. The number of giants with both $\mathrm{O}$ and $\mathrm{Na}$ abundances measured in $\mathrm{M} 80$ is 50 .

The spread in $[\mathrm{O} / \mathrm{Fe}]$ and $[\mathrm{Na} / \mathrm{Fe}]$ ratios in $\mathrm{M} 80$ is significant, about 1 dex for both elements. The resulting $\mathrm{Na}-\mathrm{O}$ anticorrelation (Fig. 7) is of moderate extension, reaching down $[\mathrm{O} / \mathrm{Fe}] \sim-0.5$ dex. This chemical signature of multiple stellar populations presents the usual features: a minority of stars with typical composition from core-collapse supernovae (high $\mathrm{O}$, low $\mathrm{Na}$ ), separated more or less clearly at $[\mathrm{Na} / \mathrm{Fe}] \sim+0.3 \mathrm{dex}$ from the bulk of stars with increasing $\mathrm{Na}$ and decreasing $\mathrm{O}$ abundances. Finally, a few stars show signatures of extreme processing: very low $\mathrm{O}$ and high $\mathrm{Na}$ levels.

The different components are clearly apparent in the distribution of $[\mathrm{O} / \mathrm{Na}]$ ratios, shown in Fig. 8: a dip between 0.0 and -0.1 dex separates the two main populations, followed by a short tail at very low $[\mathrm{O} / \mathrm{Na}]$ values.

Following the criteria defined in Carretta et al. (2009a), we can identify also in M 80 a fraction of stars with primordial $(P)$ composition, and two components of second generation with intermediate $(I)$ and extreme $(E)$ chemistry processed through hot H-burning. In M 80 the fractions we found are $P=36 \pm 8 \%$ $I=56 \pm 11 \%$, and $E=8 \pm 4 \%$, respectively. We caution that because of the limitations of the mechanical positioning of fibres, our sample is composed of stars located outside two half-mass radii from cluster centre. Since the global population fraction can be best characterised from stars between 1 and 2 half mass radii, first generation stars could be over-represented in the observed sample, as pointed out by the referee. However, we note that the $P, I$, and $E$ fractions in M 80 are not exceptional with respect to the values found in other GCs with a large variety of concentration, mass, and dynamical age (see Carretta et al. 2009a, their Table 5).

The relations among all the abundances available for the light elements $\mathrm{O}, \mathrm{Na}, \mathrm{Mg}, \mathrm{Al}$, and $\mathrm{Si}$ in $\mathrm{M} 80$ are shown in Fig. 9. Al was only available for the 14 stars observed with UVES (one upper limit, 13 detections). Our average value (+0.48 dex, $\sigma=0.34 \mathrm{dex})$ is in good agreement with that from ten stars in Cavallo et al. (2004: $+0.37 \mathrm{dex}, \sigma=0.48 \mathrm{dex})$. The $[\mathrm{Al} / \mathrm{Fe}] \mathrm{ra}-$ tios present well-defined anti-correlations with species depleted in proton-capture reactions $(\mathrm{O}$, and $\mathrm{Mg}$ ) and a neat correlation with $\mathrm{Na}$ abundances (middle panels of Fig. 9).

The other elements involved in hot H-burning $(\mathrm{Mg}, \mathrm{Si})$ do not seem to be particularly touched by nuclear processing in now extinct first generation stars. The only exception is star 13987 , observed with UVES, which shows the lowest $\mathrm{Mg}$ and $\mathrm{O}$ abundances and, accordingly, the highest $\mathrm{Al}, \mathrm{Si}$ (but not Na) abundances in our sample. This object drives the correlation between $\mathrm{Mg}$ and $\mathrm{O}$ observed in our sample of stars with UVES spectra (top-left panel in Fig. 9) as well as the correlation Si-Al and the $\mathrm{Mg}$-Si anti-correlation (top right panel and bottom right panel in Fig. 9). We conclude (with a cautionary word about the limited size of our UVES sample) that in M 80 the nuclear processing at extremely hot temperatures affected only a trace fraction of stars, at odds with what occurred in other GCs, such as NGC 4833 (Carretta et al. 2014b), a cluster with a very similar (present-day) total mass. 
Table 5. Abundances of proton-capture elements in stars of NGC 6093.

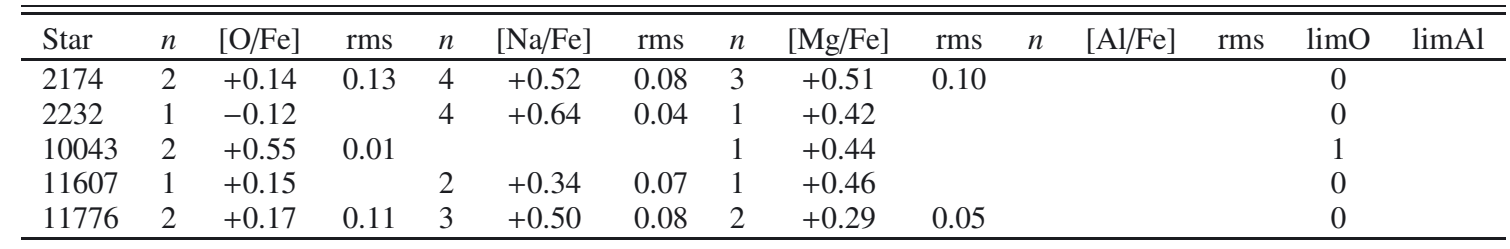

Notes. Upper limits $(\operatorname{limO}, \mathrm{Al}=0)$ and detections $(=1)$ for $\mathrm{O}$ and $\mathrm{Al}$ are flagged. The complete table is available at CDS.

Table 6. Abundances of $\alpha$-elements in stars of NGC 6093.

\begin{tabular}{lcccccccccccc}
\hline \hline Star & $n$ & {$[\mathrm{Si} / \mathrm{Fe}]$} & $\mathrm{rms}$ & $n$ & {$[\mathrm{Ca} / \mathrm{Fe}]$} & $\mathrm{rms}$ & $n$ & {$[\mathrm{Ti} / \mathrm{Fe}] \mathrm{I}$} & $\mathrm{rms}$ & $n$ & {$[\mathrm{Ti} / \mathrm{Fe}] \mathrm{II}$} & $\mathrm{rms}$ \\
\hline 2174 & 3 & +0.33 & 0.15 & 7 & +0.36 & 0.10 & 1 & +0.15 & & & & \\
2232 & 4 & +0.39 & 0.16 & 5 & +0.36 & 0.07 & 4 & +0.19 & 0.04 & & & \\
10043 & 1 & +0.36 & & 5 & +0.36 & 0.10 & 4 & +0.20 & 0.08 & & & \\
11607 & 7 & +0.35 & 0.15 & 7 & +0.36 & 0.08 & 3 & +0.18 & 0.20 & & & \\
11776 & 4 & +0.38 & 0.03 & 5 & +0.35 & 0.05 & 3 & +0.18 & 0.18 & & & \\
\hline
\end{tabular}

Notes. The complete table is available at CDS.

Table 7. Abundances of Fe-peak elements in stars of NGC 6093.

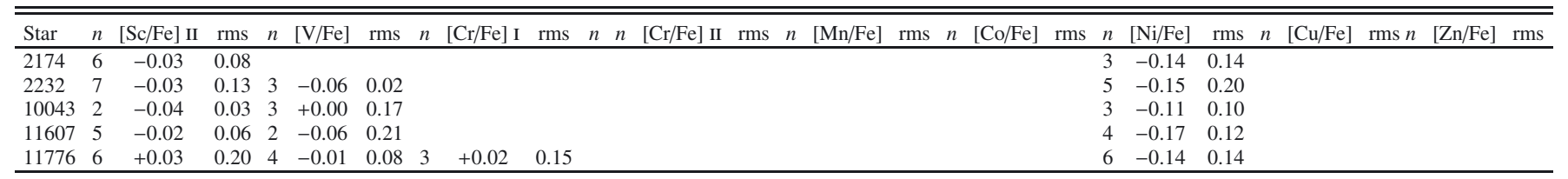

Notes. The complete table is available at CDS.

Table 8. Abundances of $n$-capture elements in stars of NGC 6093 with UVES spectra.

\begin{tabular}{|c|c|c|c|c|c|c|c|c|c|c|c|c|c|c|c|c|c|c|c|c|c|c|c|c|}
\hline Star & $n$ & {$[\mathrm{Y} / \mathrm{Fe}]$ II } & $\mathrm{rms}$ & $n$ & {$[\mathrm{Zr} / \mathrm{Fe}] \mathrm{I}$} & $\mathrm{rms}$ & $n$ & {$[\mathrm{Zr} / \mathrm{Fe}]$ II $\mathrm{rms}$} & $n$ & {$[\mathrm{La} / \mathrm{Fe}]$ II $\mathrm{rms}$} & $n$ & {$[\mathrm{Ce} / \mathrm{Fe}]$ II $\mathrm{rms}$} & $n$ & {$[\mathrm{Pr} / \mathrm{Fe}] \mathrm{II}$} & $\mathrm{rms}$ & $n$ & {$[\mathrm{Nd} / \mathrm{Fe}] \mathrm{II}$} & $\mathrm{rms}$ & $n$ & {$[\mathrm{Sm} / \mathrm{Fe}]$ II } & $\mathrm{rms}$ & $n$ & {$[\mathrm{Eu} / \mathrm{Fe}]$ II } & $\mathrm{rms}$ \\
\hline 13474 & 13 & -0.10 & 0.12 & 3 & -0.07 & 0.07 & 1 & +0.00 & 1 & +0.34 & 1 & +0.16 & 4 & +0.21 & 0.17 & 9 & +0.22 & 0.08 & 1 & +0.46 & & 2 & +0.53 & 0.04 \\
\hline 13987 & 11 & -0.04 & 0.08 & & & & 1 & +0.07 & 1 & +0.46 & 1 & +0.30 & 3 & +0.32 & 0.11 & 7 & +0.31 & 0.09 & 1 & +0.46 & & 1 & +0.52 & \\
\hline 14148 & 9 & +0.02 & 0.10 & & & & 1 & +0.00 & 1 & +0.47 & 1 & +0.41 & 4 & +0.31 & 0.15 & 6 & +0.31 & 0.09 & 1 & +0.41 & & 2 & +0.51 & 0.06 \\
\hline 14656 & 13 & -0.08 & 0.13 & 1 & +0.04 & & 1 & +0.09 & 1 & +0.42 & 1 & +0.28 & 4 & +0.34 & 0.16 & 7 & +0.35 & 0.09 & 2 & +0.39 & 0.06 & 2 & +0.49 & 0.04 \\
\hline 15308 & 12 & -0.15 & 0.08 & 3 & -0.10 & 0.04 & 1 & -0.09 & 1 & +0.20 & 1 & +0.07 & 4 & +0.10 & 0.16 & 6 & +0.11 & 0.04 & 2 & +0.32 & 0.05 & 2 & +0.48 & 0.08 \\
\hline
\end{tabular}

Notes. The complete table is available at CDS.

Table 9. Abundances of Ba II in stars of NGC 6093.

\begin{tabular}{lccc}
\hline \hline Star & $n$ & {$[\mathrm{Ba} / \mathrm{Fe}] \mathrm{II}$} & $\mathrm{rms}$ \\
\hline 2174 & 1 & +0.22 & \\
2232 & 1 & -0.04 & \\
10043 & 1 & +0.03 & \\
11607 & 1 & +0.09 & \\
11776 & 1 & +0.57 & \\
\hline
\end{tabular}

Notes. The complete table is available at CDS.

\subsection{Elements in the $\alpha$ and Fe-groups}

The run of $\alpha$-elements as a function of the temperature is shown in Fig. 6. Apart from a small increase in the scatter for $\mathrm{Mg}$, the other elements of this group ( $\mathrm{Si}, \mathrm{Ca}, \mathrm{Ti})$ are very homogeneous in $\mathrm{M} 80$ with dispersion totally explained by uncertainties in the analysis. In Fig. 6 we also plot the mean $[\alpha / \mathrm{Fe}]$ ratio obtained from the average of $[\mathrm{Si} / \mathrm{Fe}],[\mathrm{Ca} / \mathrm{Fe}]$, and $[\mathrm{Ti} / \mathrm{Fe}] \mathrm{I}:+0.308 \pm$ 0.003 dex ( $\sigma=0.026$ dex, 82 stars). Had we also included $\mathrm{Mg}$ in the mean, the value would have been $+0.342 \pm 0.004(\sigma=$ $0.032 \mathrm{dex}, 82 \mathrm{stars})$. This provides more evidence that only a few stars in M 80 were polluted by material processed at the high temperatures required for a significant depletion of $\mathrm{Mg}$.

The elements of the Fe-group do not show any intrinsic scatter in M 80 and usually track iron, apart from $\mathrm{Mn}$ and $\mathrm{Cu}$ (only measured in stars with UVES spectra), which present the usual under-abundance typical of metal-poor GCs (see Gratton et al. 2004). In cases where we measured both neutral and singly ionised transitions for a species $(\mathrm{Fe}, \mathrm{Ti}, \mathrm{Cr})$, there is excellent agreement between abundances.

\subsection{Neutron-capture elements}

We derived the abundances of a few neutron-capture elements, mainly for stars with UVES spectra, apart from Ba, for which a transition was measurable also in stars with GIRAFFE spectra and HR13 setup.

Concerning $\mathrm{Ba}$, the lines available are strong and more sensitive to velocity fields than to the abundances. To avoid the ensuing trends as a function of the $v_{\mathrm{t}}$, whose values are derived from a plaethora of weaker Fe lines, we adopted the procedure followed in Carretta et al. (2013, 2014a). Abundances of Ba were obtained using a common $[\mathrm{Fe} / \mathrm{H}]$ for all stars (equal to the mean 
A\&A 578, A116 (2015)

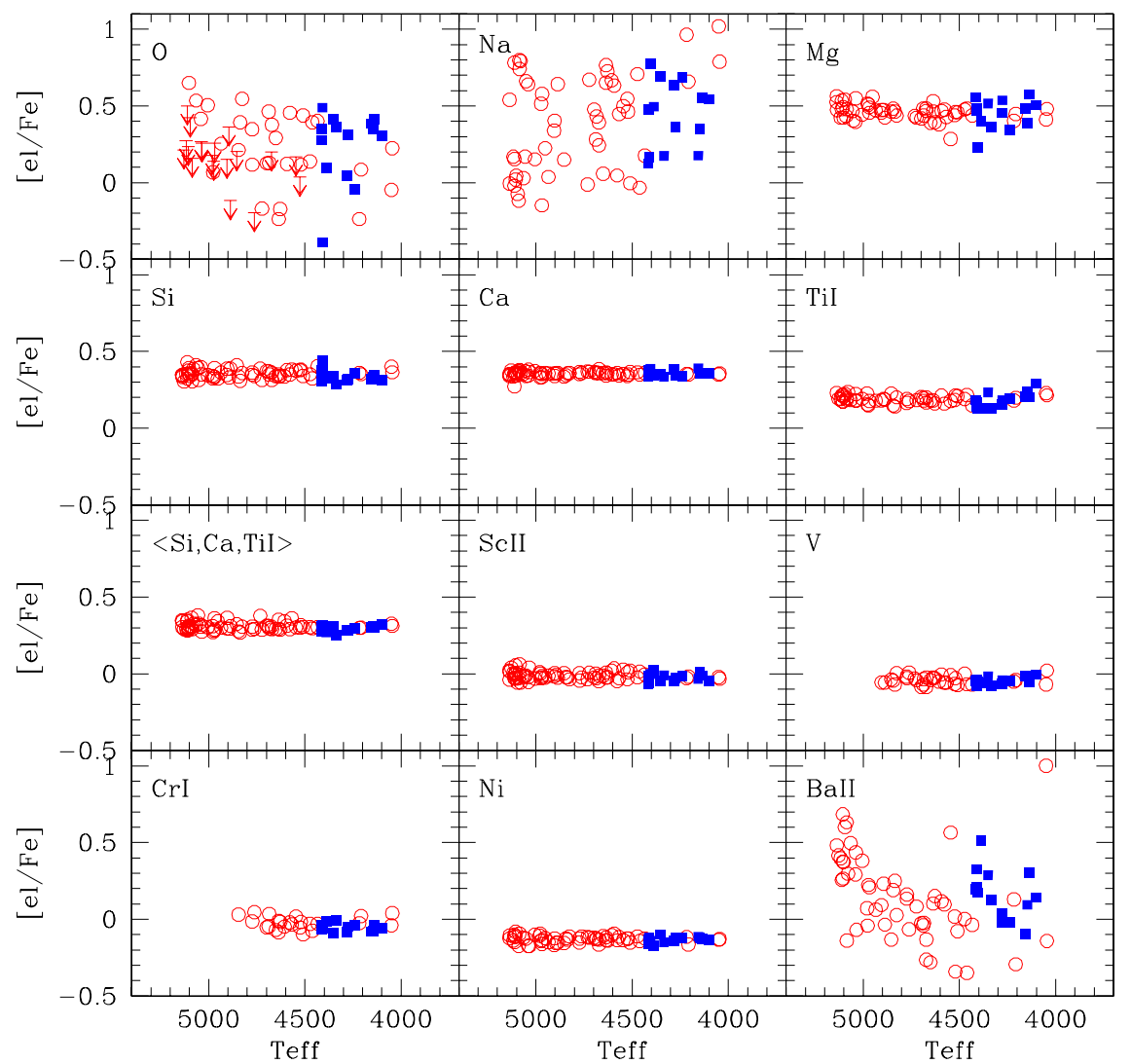

Fig. 6. Abundance ratios $[\mathrm{el} / \mathrm{Fe}]$ as a function of $T_{\text {eff }}$ for all stars in our sample. Filled squares and open circles indicate stars with UVES and GIRAFFE spectra, respectively. Internal error bars are listed in Tables A.1 and A.2.

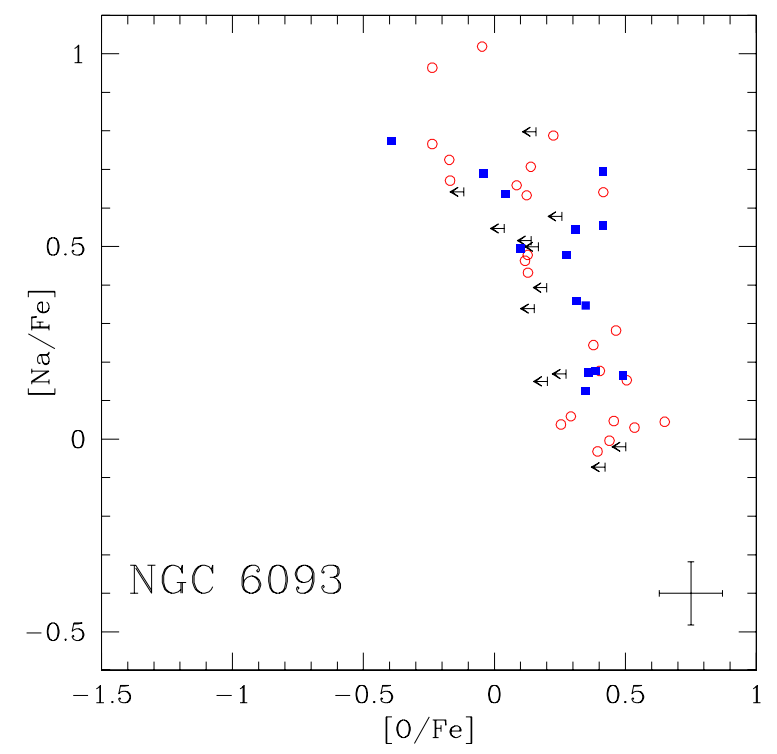

Fig. 7. Anti-correlation between $\mathrm{Na}$ and $\mathrm{O}$ abundances in $\mathrm{M}$ 80. Empty circles indicate stars observed with GIRAFFE, filled squares with UVES. Upper limits in $\mathrm{O}$ are indicated with arrows. The error bars represent internal errors (see Appendix A).

value $-1.79 \mathrm{dex}$ ) and values of $v_{\mathrm{t}}$ provided by the relation found by Worley et al. (2013). The abundances from EWs of all three $\mathrm{Ba}$ lines available in the UVES spectra were checked with synthetic spectra employing the line lists by D'Orazi et al. (2012), finding good agreement between the two methods. The results are shown in the last panel of Fig. 6 as a function of temperature: most of the observed spread is accounted for with uncertainties derived from the analysis (see error tables in Appendix A).

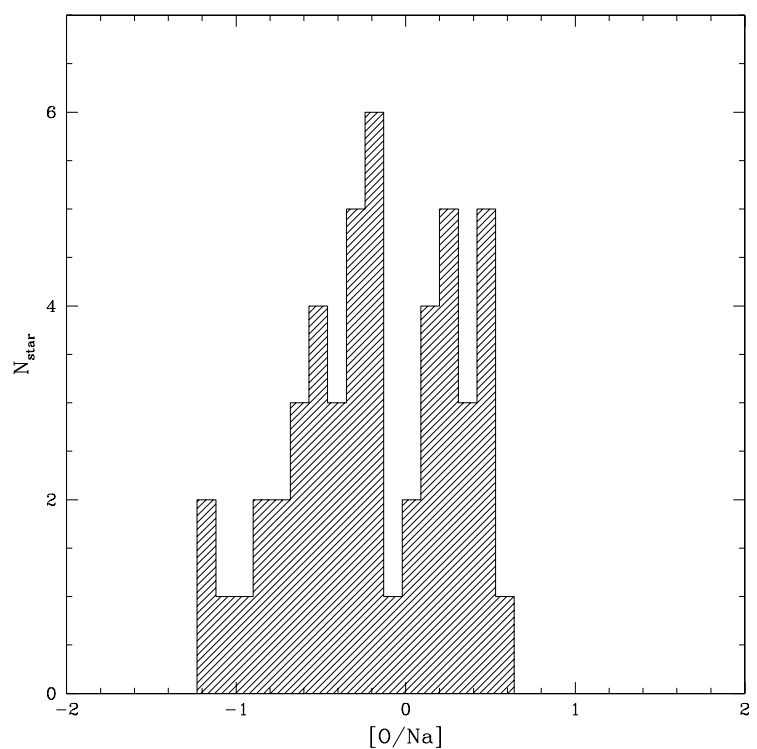

Fig. 8. Distribution of the $[\mathrm{O} / \mathrm{Na}]$ ratios in $\mathrm{M} 80$.

The abundances of $\mathrm{Ba}$ are not correlated with any of the protoncapture elements.

Star $16162\left(\right.$ with $\left.T_{\text {eff }}=4050 \mathrm{~K}\right)$ stands out with its $[\mathrm{Ba} / \mathrm{Fe}]=$ 1.001 dex, the highest in all our sample. This star has also the highest $\mathrm{Na}$ abundance and it is characterised by the reddest $B-V$ colour in our data set of stars. In Fig. 10 the spectrum of this Ba-rich star is compared in the region of the Ba II $6141 \AA$ line to the spectra of the three stars with most similar temperatures (and much lower Ba abundances, see Table 9). The spectral lines of star 16162 show a clear broadening with respect to the other stars. We conclude that this object likely presents 

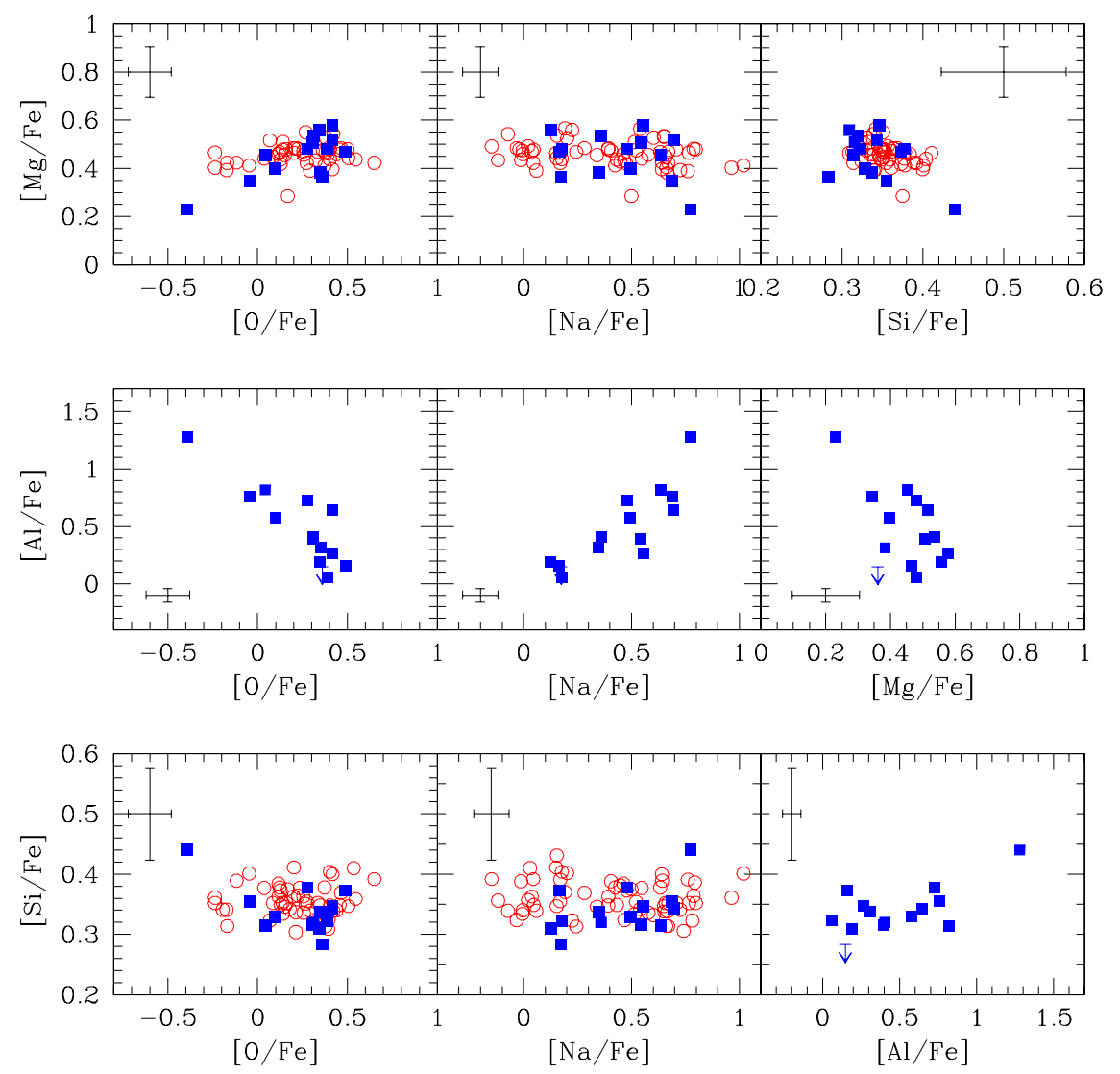

Fig. 9. Relations among the abundances of protoncapture elements in red giants of M 80. Filled squares and empty circles indicate stars observed with UVES and GIRAFFE, respectively. Internal error bars are also shown.

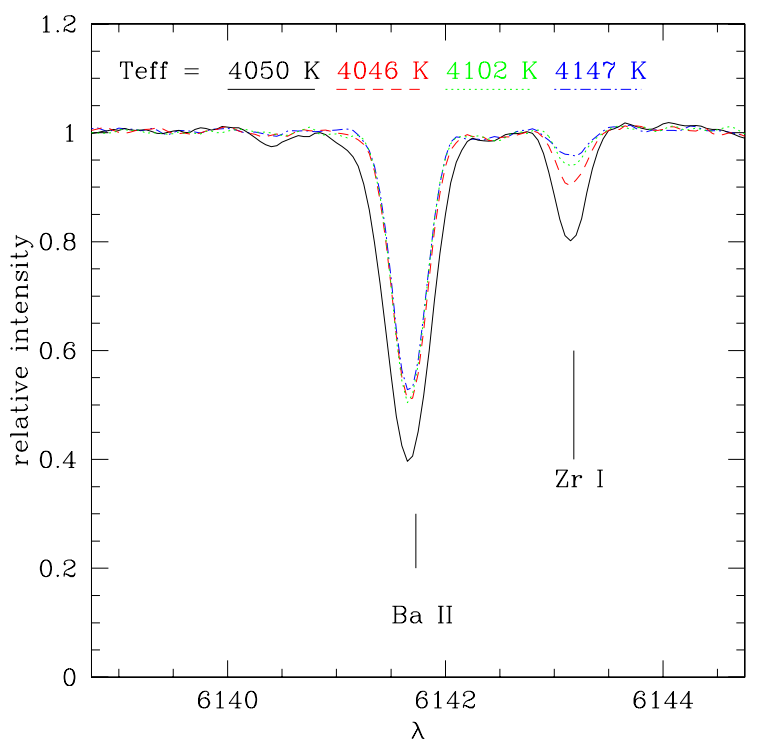

Fig. 10. GIRAFFE spectrum of star $16162\left(T_{\text {eff }}=4050 \mathrm{~K},[\mathrm{Ba} / \mathrm{Fe}]=\right.$ +1.001 dex) in the region of the Ba II $6141 \AA$ line (solid line) compared with stars with similar temperatures: 14224 (dashed line), 17886 (dotted line), and 17874 (dot dashed line).

hints of a phenomenon of mass transfer from a binary companion, although its RV does not show particular differences with respect to other stars. Anyway, by eliminating this star the average from GIRAFFE spectra changes only a little, becoming $[\mathrm{Ba} / \mathrm{Fe}]=+0.11 \pm 0.04 \operatorname{dex}(\sigma=0.25$ dex, 58 stars $)$.

The results for Y II were validated through spectral synthesis of the strong line at $4883 \AA$, using a line list from D'Orazi et al. (2013). We obtained La and Ce abundances from the lines at $6390 \AA$ and at $5274 \AA$, using spectrum synthesis and EWs, respectively, whereas Pr (4 lines) and Sm (2 lines) were analysed using atomic parameters from Sneden et al. (2003) and Koch \& McWilliam (2014). Abundances of Nd were derived as in Carretta et al. (2011b).

\section{Discussion: $\mathbf{M} 80$ in context}

\subsection{Multiple stellar populations in $M 80$}

The spectroscopic observations in M 80 complete the core sample of our FLAMES survey of the $\mathrm{Na}-\mathrm{O}$ anti-correlation in Galactic GCs. We studied 24 GCs (about $15 \%$ of those listed in the Harris 1996 catalog): the range of main parameters covered by our sample is reported in Table 10, together with the values for M 80.

M 80 is a typical inner halo GC (Carretta et al. 2010a), and also the chemistry of its multiple stellar populations appears to be normal. The extension of the Na-O anti-correlation, the most prominent signature of multiple stellar generations, is quantified by a IQR $[\mathrm{O} / \mathrm{Na}]=0.784$. This value locates $\mathrm{M} 80$ right in the middle of the main relations defined by other GCs in our core sample, as the correlation between extent of the anti-correlation and total cluster mass (upper panel of Fig. 11).

In Carretta et al. (2014b) we discovered a tight relation (once the post-core collapse GCs are excluded) linking the cluster concentration and the residuals around the relation between $M_{V}$ and $\mathrm{IQR}[\mathrm{O} / \mathrm{Na}]$. More concentrated clusters seem to develop a less extended $\mathrm{Na}-\mathrm{O}$ anti-correlation with respect to more loose GCs. The anti-correlation of M 80 is in very good agreement with this trend (Fig. 11, bottom panel).

In M 80 we were able to observe a number of stars below the bump level on the luminosity function of the RGB $(V=16.12 \pm 0.03 \mathrm{mag}$, Zoccali et al. 1999). As predicted by 
Table 10. Summary of the global parameters sampled in GCs of our FLAMES survey.

\begin{tabular}{|c|c|c|c|}
\hline Parameter & Min & Max & NGC 6093 \\
\hline$M_{V}(\mathrm{mag})$ & $\begin{array}{r}-6.64 \\
\text { NGC } 6397\end{array}$ & $\begin{array}{r}-9.98 \\
\text { NGC } 6715\end{array}$ & -8.23 \\
\hline$R_{\mathrm{GC}}(\mathrm{kpc})$ & $\begin{array}{r}3.1 \\
\text { NGC } 6388\end{array}$ & $\begin{array}{r}18.9 \\
\text { NGC } 6715\end{array}$ & 3.8 \\
\hline$[\mathrm{Fe} / \mathrm{H}](\mathrm{dex})$ & $\begin{array}{r}-2.34 \\
\text { NGC } 7099\end{array}$ & $\begin{array}{r}-0.43 \\
\text { NGC } 6441\end{array}$ & -1.79 \\
\hline$c$ & $\begin{array}{r}0.93 \\
\text { NGC } 6809\end{array}$ & $\begin{array}{r}2.29 \\
\text { NGC } 7078\end{array}$ & 1.68 \\
\hline $\log t_{c}(\mathrm{yr})$ & $\begin{array}{r}4.94 \\
\text { NGC } 6397\end{array}$ & $\begin{array}{r}8.99 \\
\text { NGC } 288\end{array}$ & 7.78 \\
\hline$\rho_{0}\left(L_{\odot} / \mathrm{pc}^{3}\right)$ & $\begin{array}{r}1.78 \\
\text { NGC } 0288\end{array}$ & $\begin{array}{r}5.76 \\
\text { NGC } 6397\end{array}$ & 4.79 \\
\hline$r_{\mathrm{h}}(\mathrm{pc})$ & $\begin{array}{r}1.56 \\
\text { NGC } 6397\end{array}$ & $\begin{array}{r}5.68 \\
\text { NGC } 0288\end{array}$ & 1.89 \\
\hline$r_{\mathrm{t}}(\mathrm{pc})$ & $\begin{array}{r}10.43 \\
\text { NGC } 6838\end{array}$ & $\begin{array}{r}90.02 \\
\text { NGC } 4590\end{array}$ & 38.63 \\
\hline HB index & $\begin{array}{r}-1.00 \\
\text { NGC } 6388\end{array}$ & $\begin{array}{r}1.00 \\
\text { NGC } 6752\end{array}$ & 0.93 \\
\hline Rel. age & $\begin{array}{r}0.84 \\
\text { NGC } 0362\end{array}$ & $\begin{array}{r}1.06 \\
\text { NGC } 7099\end{array}$ & 1.03 \\
\hline $\mathrm{IQR}[\mathrm{O} / \mathrm{Na}]$ & $\begin{array}{r}0.257 \\
\text { NGC } 6838\end{array}$ & $\begin{array}{r}1.169 \\
\text { NGC } 6715\end{array}$ & 0.784 \\
\hline
\end{tabular}

Notes. $M_{V}, R_{\mathrm{GC}}, c, \log t_{\mathrm{c}}, \rho_{0}$ from Harris (1996, 2010 edition); $[\mathrm{Fe} / \mathrm{H}]$ from Carretta et al. (2009c, 2010b, 2011b, 2013, 2014b); $c$ : excluding post-core collapse GCs (NGC 6397,6752,7099), where $c=2.5 ; r_{\mathrm{h}}, r_{\mathrm{t}}$, HB index: Mackey \& Van den Bergh (2005); relative age: Carretta et al. (2010a); IQR[O/Na]: our FLAMES survey.

Salaris et al. (2006), the luminosity of the RGB bump is sensitive to the He abundance (the bump is brighter for a population where a He-enhanced component is present). This prediction has been long since observationally confirmed both with spectroscopy (using the component with $\mathrm{Na}$ enhancement in GCs as a proxy, see Carretta et al. 2007a; Bragaglia et al. 2010) and with photometry (e.g. Nataf et al. 2011). In Fig. 12 we show the cumulative distribution of the magnitude differences $V-V_{\text {bump }}$ for all stars in M 80 with a measured $\mathrm{Na}$ abundance, separating the first generation stars ( $P$ component) from second generation stars $(I+E$ components $)$.

Although a Kolmogorov-Smirnov test formally does not allow us to reject the null hypothesis that the two subsamples are extracted from the same parent distribution, it is clear that regardless of adopted magnitude cut the second generation stars are on average located at slightly brighter luminosities than the first generation giants, as they should be according to their He-enhanced abundances.

In our present sample of giants in M 80, we did not find a statistically significant difference in radial distribution. However, also taking the physical limitations imposed by the fibres positioning tool into account, a more suitable approach to study the radial concentration of different stellar generations is offered
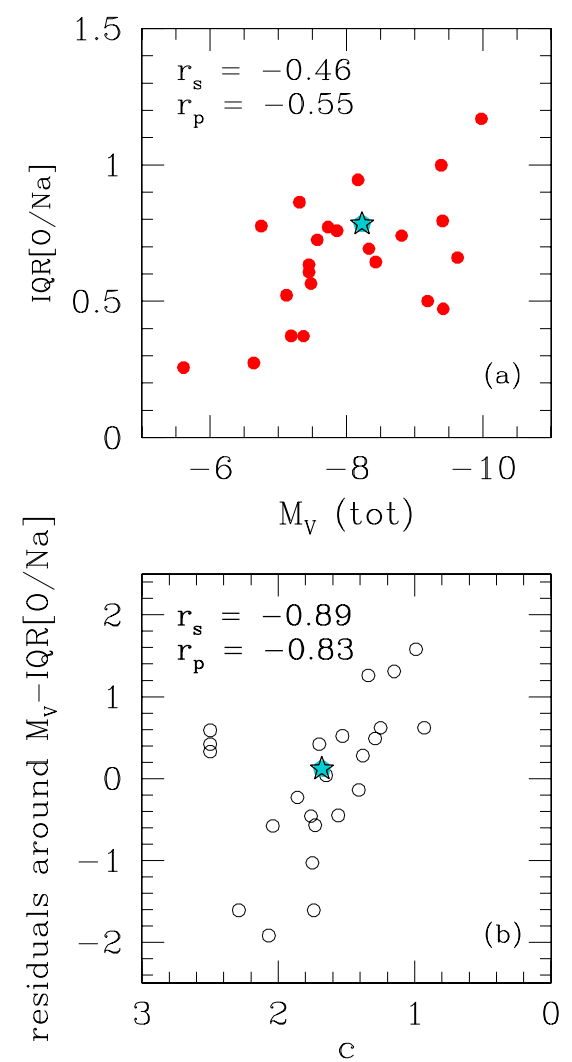

Fig. 11. Upper panel: correlation between total absolute magnitude and the interquartile range of the $[\mathrm{O} / \mathrm{Na}]$ ratio for $\mathrm{GC}$ in our FLAMES survey. Lower panel: relation between cluster concentration and the residual around the best-fit relation IQR $[\mathrm{O} / \mathrm{Na}]-M_{V}($ tot $) . \mathrm{M} 80$ is indicated by a filled star symbol. In each panel the Spearman rank correlation coefficient $r_{\mathrm{s}}$ and the Pearson linear correlation coefficient $r_{\mathrm{p}}$ are listed.

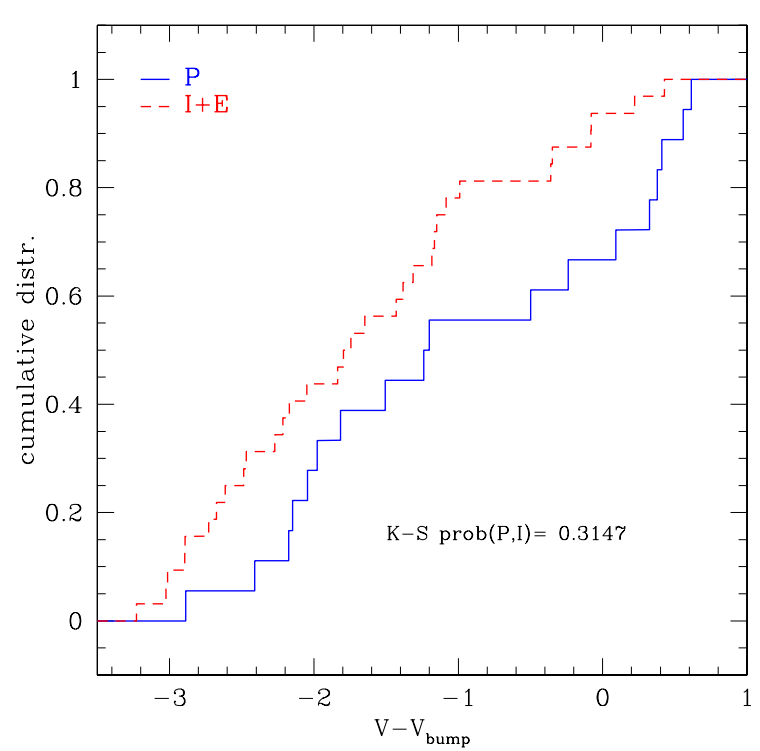

Fig. 12. Cumulative distribution of the difference in magnitude between individual $V$ and $V_{\text {bump }}=16.12 \mathrm{mag}$ for all stars of the first generation (solid line) and of the second generation (dashed line) with measured $\mathrm{Na}$ abundances.

by photometry of large samples over an extended area on the cluster. The combination $(U-B)-(B-I)$, which Monelli et al. (2013) call $c_{U, B, I}$, is a good way to study the separation of 


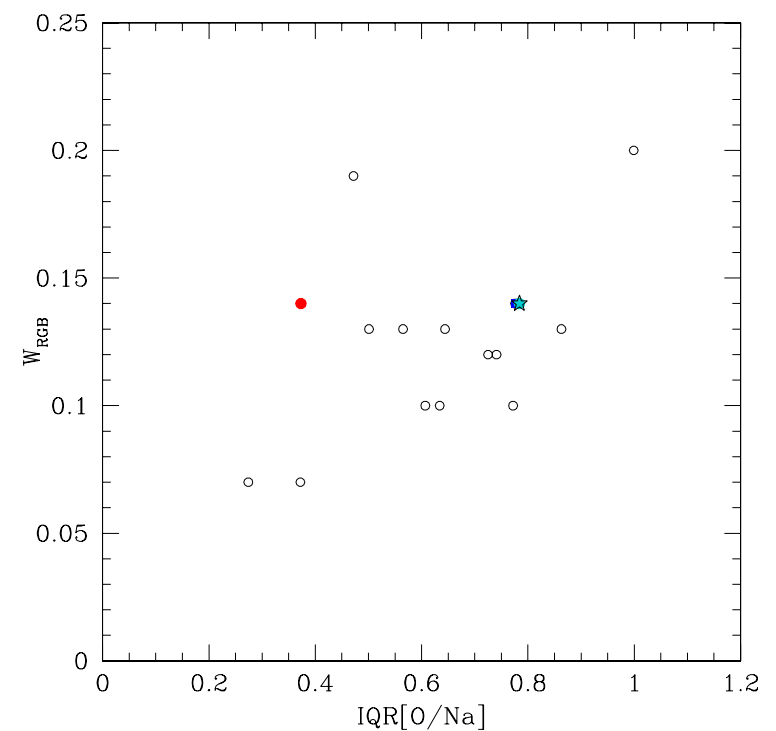

Fig. 13. Width of the RGB from the SUMO survey $\left(W_{\mathrm{RGB}}\right)$ as a function of the interquartile range IQR[O/Na] from our FLAMES survey for the 16 GCs analysed in both samples. M 80 is indicated with a star symbol, under which NGC 288 (blue filled square) is almost hidden. M 4 is indicated with a filled red circle.

stars according to different populations in a GC, since it does not significantly depend on reddening and distance modulus. There are 16 objects, including M 80, in common between the SUMO sample and our FLAMES survey. Unfortunately the photometry of individual stars is still unpublished, but Monelli et al. provide the width $W_{\mathrm{RGB}}$ of the RGB in $c_{U, B, I}$, which can be used to better investigate the relation between spectroscopic and photometric properties of multiple stellar populations in M 80 and in other GCs (see Fig. 13).

For M 80 they derive a value $W_{\mathrm{RGB}}=0.14$, which is the same width observed for NGC 288 and M 4 (NGC 6121). This occurrence is clearly at odds with the behaviour of these GCs concerning the $\mathrm{Na}-\mathrm{O}$ anti-correlation, as shown in the upper panels of Fig. 14, where we plot the $[\mathrm{Na} / \mathrm{Fe}]$ and $[\mathrm{O} / \mathrm{Fe}]$ ratios of each cluster from our strictly homogeneous FLAMES survey.

In this figure we indicate with a vertical line the lowest border of the $\mathrm{O}$ abundance measured in $\mathrm{M}$ 80, and superimposed this border to the other two plots. The Na-O anticorrelation derived for $\mathrm{M} 4$ (with $\mathrm{IQR}[\mathrm{O} / \mathrm{Na}]=0.373)^{4}$ is clearly much shorter than those observed in both M 80 and NGC 288 (IQR $=0.784$ and 0.776 , respectively). Therefore it is not a surprise that $W_{\mathrm{RGB}}$ and IQR[O/Na] are not well correlated (see Fig. 13). The Pearson's linear correlation coefficient is $r=0.46$, which for $16 \mathrm{GCs}$ indicates that the correlation is significant only at a level of confidence between 90 and $95 \%$.

These three GCs are different, however, in other relevant global parameters: the total metallicity decreases going from M 4 to NGC 288 and to $\mathrm{M} 80$ ([Fe/H $=-1.17,-1.30,-1.79$ dex, respectively, from high-resolution UVES spectra, Carretta et al. 2009c, and this work), and the HB morphology significantly differs among these clusters, as evident from the CMDs from the ACS survey (Sarajedini et al. 2007) plotted in the lower panels of Fig. 14. The HB morphological index (defined as $(B-R) /(B+V+R)$ from the number of stars $B, V, R$ bluer

\footnotetext{
4 We used the values derived from our homogeneous work, but other independent studies also support evidence that the $\mathrm{Na}-\mathrm{O}$ anticorrelation is not very extended in M 4 (see e.g. Marino et al. 2008).
}

than, inside, and redder than the instability strip, Lee 1990) is $0.93,0.98$, and -0.06 for NGC 288, M 80, and M 4, respectively (Mackey \& van den Bergh 2005), corresponding to the differences clearly visible in the CMDs of the lower panels in Fig. 14. The HB stellar distribution in the first two GCs is all to the blue of the RR Lyrae instability strip, starting almost at the same colour, but reaching far more fainter (hotter) levels in M 80 than in NGC 288. On the other hand, the blue part of the HB in M 4 barely ends at the colour where the HB distribution starts in the two other GCs.

To better understand the missing piece of information from Fig. 13 we then computed the residuals about the relation between $W_{\text {RGB }}$ and IQR[O/Na]. These value are shown in Fig. 15 as a function of the HB index and metallicity, and allow us to better understand the difference between photometric and spectroscopic properties of multiple populations. The residuals are correlated with $\mathrm{HB}$ index and $[\mathrm{Fe} / \mathrm{H}]$, which is the first parameter controlling the star distribution on the HB: both relations are found statistically significant at a level of confidence of about $99 \%$. We conclude that star-to-star abundance variations in the lightest proton-capture elements, like $\mathrm{C}$ and $\mathrm{N}$, are easily revealed as enlargements in the photometric sequences in the CMDs, through the impact of variations in the abundance of $\mathrm{N}$, and, in particular, on the absorption molecular bands in the ultraviolet or most bluer filters. These variations also include contributions not due to multiple populations but to the standard evolution of population II low-mass stars: the first dredge-up and the second mixing episode after the RGB bump both concur to enhance the $\mathrm{N}$ abundance, equally in first and second generation stars. From the study of Monelli et al. (2013), at face value the changes in the abundances of $\mathrm{C}$ and $\mathrm{N}$ are comparable in extent for GCs, such as M 80 and M 4 and NGC 288.

Species such as $\mathrm{O}$ and $\mathrm{Na}$ cannot heavily affect the broadband filters, and this explain the somewhat loose correlation in Fig. 13. Second generation stars with O-poor/Na-rich composition are also expected to show $\mathrm{N}$ enhancement (including the contribution from mere evolutive mixing), and this gives the correlation with the width of the RGB in index like $c_{U, B, I}$. On the other hand, when $\mathrm{O}$ and $\mathrm{Na}$ are more changed with respect to the primordial composition of stars, He is also more and more modified. Taking this occurrence into account results in a tighter correlation between photometric and spectroscopic index, and produces the significant correlation with HB morphology for which $\mathrm{He}$ is the third parameter (see Gratton et al. 2010 and references therein). As a sanity check, if $\mathrm{O}$ and $\mathrm{Na}$ are more related to $\mathrm{He}$ and HB morphology, we should expect that M 80 is an example of the tight correlation between the extension of the Na-O anti-correlation and the bluest and hottest point reached along the HB, discovered by Carretta et al. (2007b). This is just the case, as shown in Fig. 16, where we used for M 80 the value $\log T_{\text {eff }}^{\max }=4.477$ from Recio-Blanco et al. (2006). M 80 nicely falls on the relation defined by the strict correspondence between the phenomenon of multiple stellar populations in GCs and their HB morphology.

\subsection{Other elements}

Elements produced with $\alpha$-capture in explosive nucleosynthesis of core-collapse supernovae $(\mathrm{Si}, \mathrm{Ca}, \mathrm{Ti})$ track each other in M 80. The observed scatter in $\mathrm{Mg}$ is from two to three times larger than that of the other $\alpha$-elements because $\mathrm{Mg}$ is also involved in the proton-capture reactions, which change the chemical composition of second generation stars with respect to the level established by Type II SNe. 

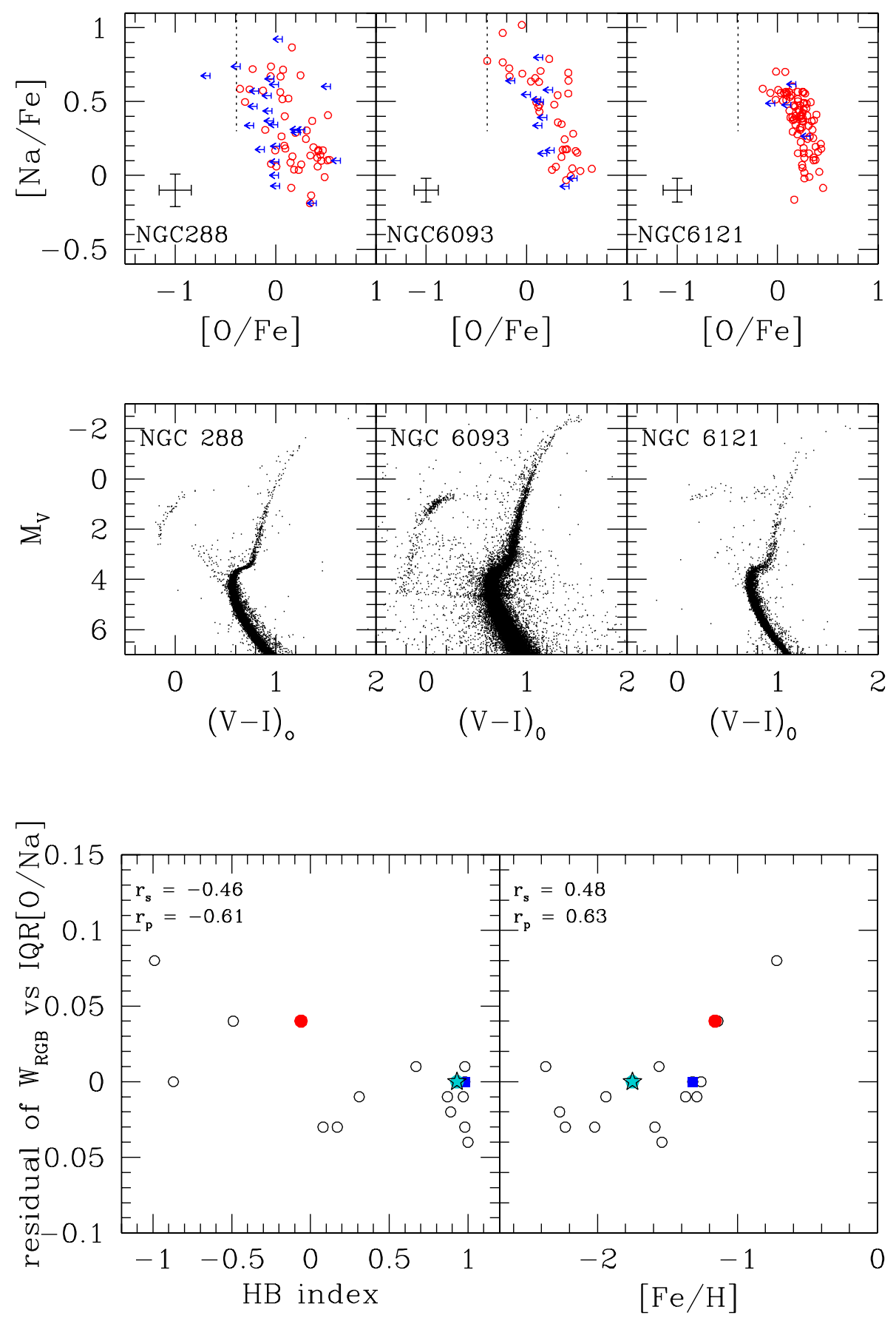

Fig. 14. Upper panels, from left to right: $\mathrm{Na}-\mathrm{O}$ anti-correlation for NGC 288 (Carretta et al. 2009a,b), M 80 (this work), and M 4 (NGC 6121, Carretta et al. 2009a,b). The vertical dotted line indicates the reach of the lowest $[\mathrm{O} / \mathrm{Fe}]$ ratio measured in $\mathrm{M} 80$, and it is superimposed to the two other GCs for comparison. Lower panels: $M_{V}$ vs. $V-I$ CMDs for the three GCs from the ACS survey by Sarajedini et al. (2007). Distance moduli and reddening values are from Harris (1996).
Fig. 15. Residuals of the $W_{\mathrm{RGB}}$ vs. IQR[O/Na] relation as a function of the $\mathrm{HB}$ index and $[\mathrm{Fe} / \mathrm{H}]$ ratio for the $16 \mathrm{GCs}$ in common with the SUMO survey. Symbols are as in Fig. 13.
The pattern of Fe-group elements in M 80 is not unusual; these elements track Fe nicely, apart from $\mathrm{Mn}$ and $\mathrm{Cu}$, which are found to be under-abundant in excellent agreement with the measurements in field stars of the Galactic halo (see Fig. 17).

We conclude that concerning the abundance pattern of species from proton- and $\alpha$-capture, as well as those of the Fepeak, M 80 is a typical halo cluster.

Also the pattern of the abundances of neutron-capture elements looks normal in M 80. In Fig. 18 we show the average abundance ratios of a light neutron-capture element, Y, of the heavier element $\mathrm{Ba}$, created by the main $s$-process, and of the Eu forged almost totally in the $r$-process (Arlandini et al. 1999). The mean ratios in M 80 are found to be compatible to the run of these elements in field stars of the Galactic halo as a function of metallicity (Venn et al. 2004). We do not confirm the very high value $[\mathrm{Eu} / \mathrm{Fe}]=+0.80$ dex found by Cavallo et al. (2004), which made M 80 outstanding in the plot showed by Venn et al.

To characterise the modality of enrichment in heavy elements from neutron capture it is safer to use the La abundance rather than $\mathrm{Ba}$ : although derived for the limited sample of stars with UVES spectra, La lines are weak and less sensitive to uncertainty in the abundance analysis. The comparison between $\mathrm{La}$ (a species with predominance of the $s$-process in the solar system) and Eu (almost totally $r$-process element in solar system) allows us to study the aforementioned enrichment. We found in M 80 a mean value of $\log \epsilon(\mathrm{La} / \mathrm{Eu})=0.44 \operatorname{dex}(\sigma=0.11 \mathrm{dex}$, 14 stars), which would indicate a predominance from $r$-process production in this cluster (pure $r$ level is $0.09 \mathrm{dex}$, Simmerer et al. 2004). 


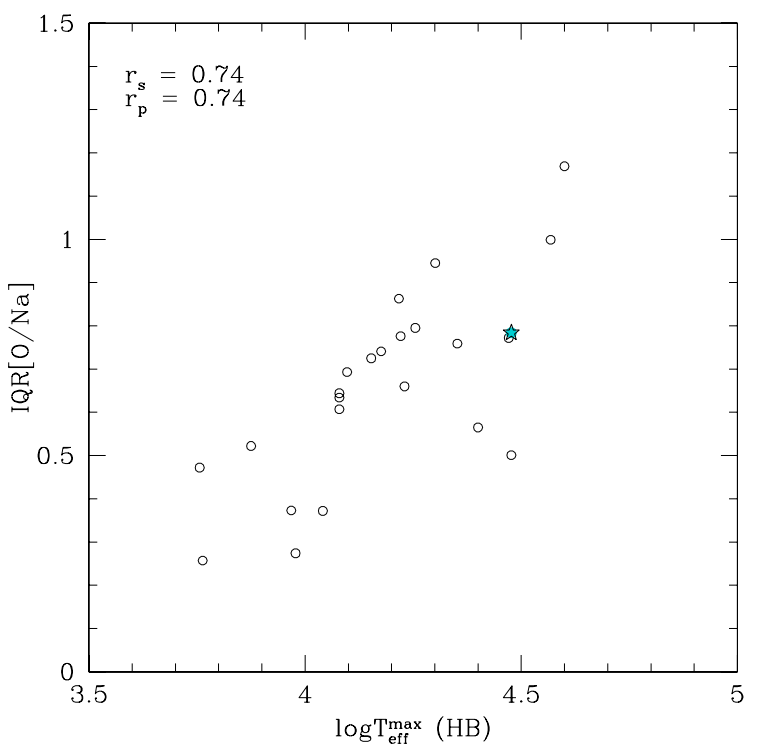

Fig. 16. Extension of the Na-O anti-correlation (measured using $\mathrm{IQR}[\mathrm{O} / \mathrm{Na}]$ ) as a function of the maximum temperature along the $\mathrm{HB}$, taken from Recio-Blanco et al. (2006). M 80 is represented by a filled star symbol. The Spearman rank and the Pearson linear correlation coefficients are listed.

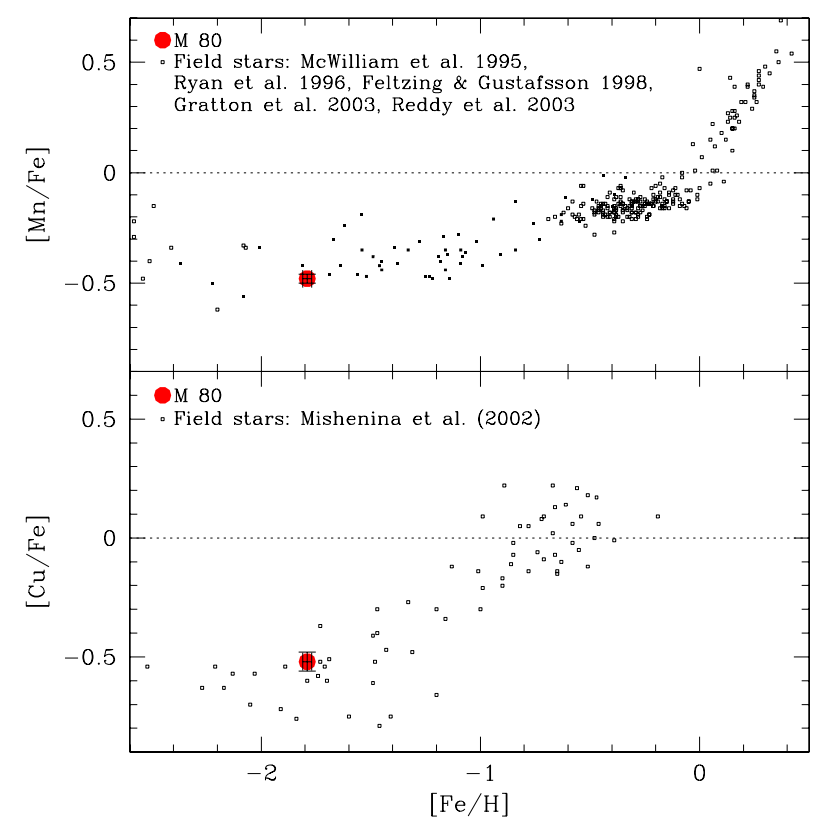

Fig. 17. $[\mathrm{Mn} / \mathrm{Fe}]$ (upper panel) and $[\mathrm{Cu} / \mathrm{Fe}]$ (lower panel) average ratios of M 80 from stars with UVES spectra compared with various samples of field stars as a function of metallicity. The root-mean square (rms) scatter for $\mathrm{Mn}, \mathrm{Cu}$, and $\mathrm{Fe}$ are plotted for M 80 (they are inside the large symbol).

A more detailed comparison can be performed by comparing all the available ratios of neutron-capture elements in M 80 with the pattern of solar scaled contributions from $s$ - and $r$-process nucleosynthesis. In the upper panel of Fig. 19, we compare the ratios with the fractions estimated in Simmerer et al. (2004). Several elements occupy a position intermediate between the pure $s$-process level and the pure $r$-fraction (obtained as usual from $N_{\mathrm{SS}, \mathrm{r}} \equiv N_{\mathrm{SS}, \text { total }}-N_{\mathrm{SS}, \mathrm{s}}$ ).

Therefore, we tried to obtain a best fit by trying to reproduce the composition of Table 4 as the sum of two contributions

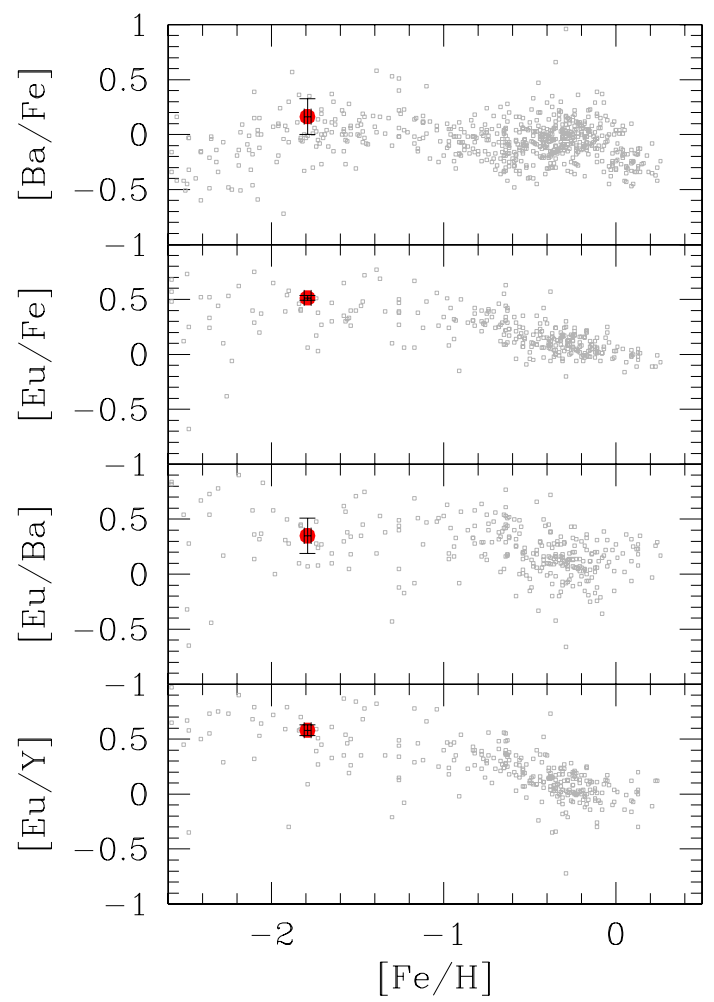

Fig. 18. From top to bottom: $[\mathrm{Ba} / \mathrm{Fe}],[\mathrm{Eu} / \mathrm{Fe}],[\mathrm{Eu} / \mathrm{Ba}]$, and $[\mathrm{Eu} / \mathrm{Y}]$ abundance ratios in $\mathrm{M} 80$ (large filled circle) and in Galactic field stars (Venn et al. 2004, empty squares) as a function of metallicity.

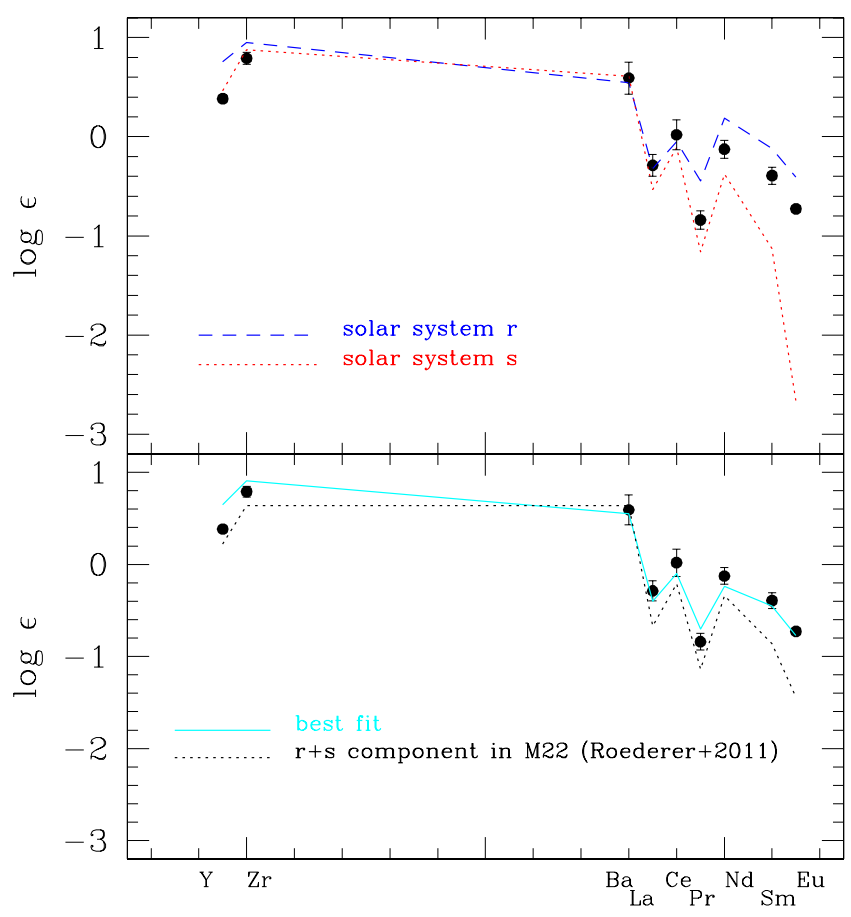

Fig. 19. Average abundances of neutron-capture elements $\mathrm{Y}, \mathrm{Zr}$, Ba, $\mathrm{La}, \mathrm{Ce}, \mathrm{Pr}, \mathrm{Nd}, \mathrm{Sm}$, and $\mathrm{Eu}$ in $\mathrm{M}$ 80. The associated rms scatters are also shown. In the upper panel, the solar scaled abundances of pure $r$-process (dashed line) and of pure $s$-process (dotted line) from Simmerer et al. (2004) are shown. In the lower panel, the attempt to best fit observed abundances with solar scaled pure $r$-process and $s$-process abundances (Simmerer et al. 2004) is shown (light blue line): the scaling factor adopted are -1.27 dex and -1.81 dex for the $r$-and $s$-process, respectively. The black dotted line connects the abundances in the $r+s$ group of the GC M 22 derived by Roederer et al. (2011). 


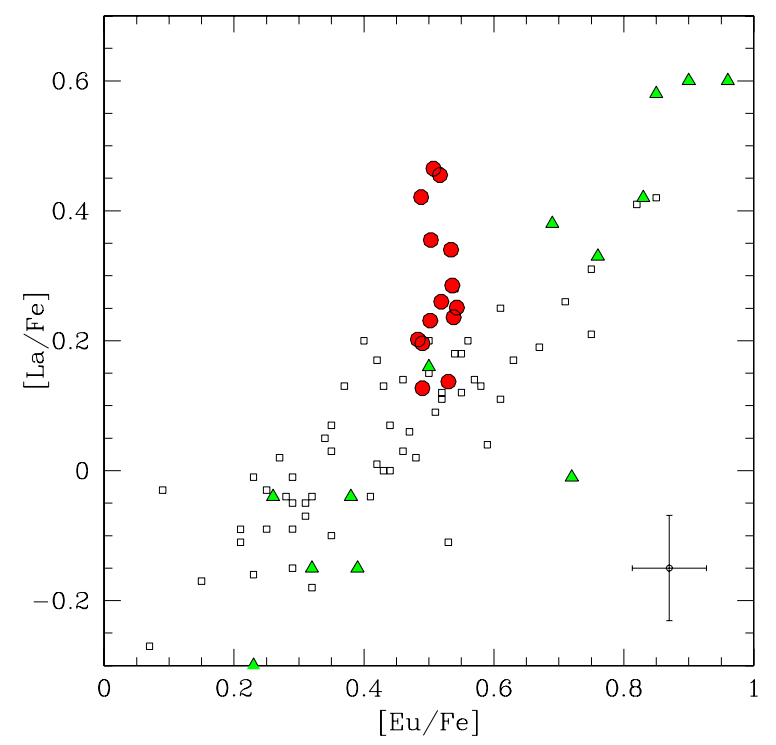

Fig. 20. $[\mathrm{La} / \mathrm{Fe}]$ ratios as a function of $[\mathrm{Eu} / \mathrm{Fe}]$ ratios for individual stars in M 80 (large filled circles) compared to $r$-only field stars from Roederer et al. (2010; small empty squares) and to a GC with a dispersion in $r$-process from Roederer (2011): M 15 (filled triangles), with abundances from Otsuki et al. (2006) and Sobeck et al. (2011). The internal error bars refer to our analysis of M 80.

(a pure solar-scaled $r$-process and a pure solar scaled $s$-process: $s$ - and $r$-fractions are as in Simmerer et al. 2004) with suitable scaling factors. We found that the observed pattern can be reproduced by using scaling factors of -1.27 dex and -1.81 dex for the $r$ - and $s$-process, respectively (see lower panel of Fig. 19). Hence, if we consider that $[\mathrm{Fe} / \mathrm{H}]=-1.79 \mathrm{dex}$, this implies $[r / \mathrm{Fe}]=+0.52$ and $[s / \mathrm{Fe}]=-0.02 \mathrm{dex}$. The excess of elements produced by the $r$-process is similar to that obtained for the $\alpha$-elements and can be interpreted as an Fe deficiency because of the fact that there was no significant contribution of SN Ia to the material from which M 80 formed. On the other hand, a significant contribution by the $s$-process is required to explain observations. The abundance ratio first/second peak of the $s$-process is more favourable to the second. This is not unexpected, given the low metallicity of M80.

To get more insight with a direct, model independent approach, we compared the pattern found in M 80 with that of two other GCs, one showing a dispersion in the $r$-process content and another GC in which a clear dispersion in $s$-process elements has been detected, chosen from the extensive survey by Roederer (2011).

In Fig. 20 our ratios $[\mathrm{Eu} / \mathrm{Fe}]$ and $[\mathrm{La} / \mathrm{Fe}]$ for individual stars in M 80 are compared to the sample of field stars with no detectable $s$-process contribution analysed by Roederer et al. (2010). Superimposed to these, we also plot stars in M 15, a GC well known to show a dispersion in the $r$-process content (see Roederer 2011). For M 15, we used data from Otsuki et al. (2006) and Sobeck et al. (2011).

The M 15 data show the same correlation between [La/Fe] and $[\mathrm{Eu} / \mathrm{Fe}]$ present in the sample of bona fide $r$-only metal-poor field stars from the compilation of Roederer et al. (2010). A dispersion in $[\mathrm{La} / \mathrm{Fe}]$ was found to be intrinsic to this sample, indicating that these variations are intrinsically associated with the $r$-process yields (Roederer et al. 2010). On the other hand, it is immediately evident that no intrinsic dispersion in $\mathrm{Eu}$ is present among giants in M 80, whereas we cannot exclude a possible small spread of La abundances in these stars.

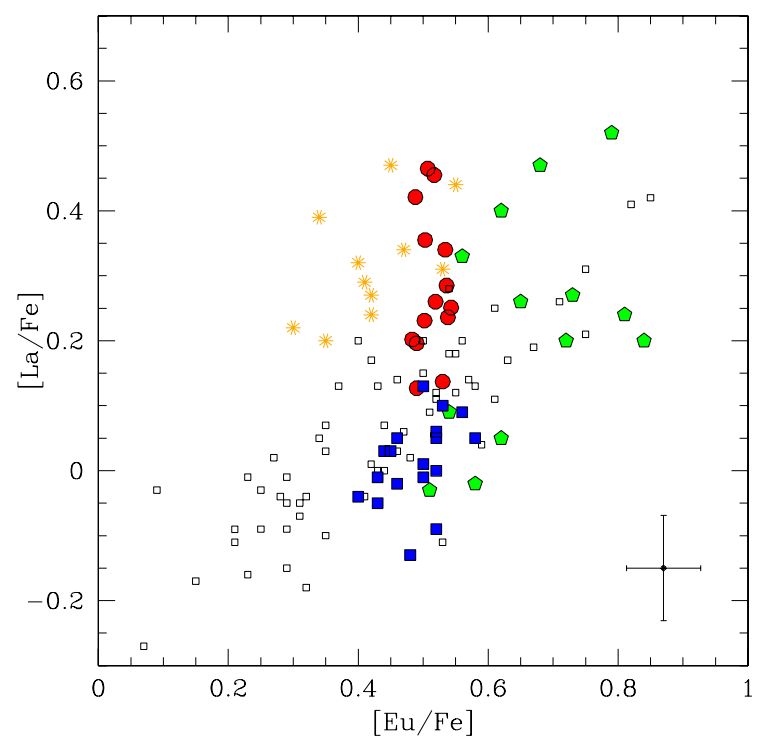

Fig. 21. $[\mathrm{La} / \mathrm{Fe}]$ ratios as a function of $[\mathrm{Eu} / \mathrm{Fe}]$ ratios for individual stars in M 80 (large filled circles) compared to $r$-only field stars from Roederer et al. (2010; small empty squares) and to GCs with a dispersion in $s$-process elements. Blue-filled squares indicate the $r$-only component in M 22 from Marino et al. (2011), orange asterisks are used for the $r+s$ component in M 22 (see Roederer et al. 2011; Marino et al. 2011), and green-filled pentagons are giants in NGC 1851 from Carretta et al. (2011b). The internal error bars refer to our present analysis of M 80.

In Fig. 21 we compare abundances of La and Eu in M 80 with those of GCs known to host stellar populations with an intrinsic dispersion in $s$-process elements: M 22 (Marino et al. 2011; Roederer et al. 2011) and NGC 1851 (Carretta et al. 2011b). The $r$-only component in M 22 (in the terminology by Roederer et al. 2011) nicely falls on the locus of galactic field metal-poor stars. This behaviour is also shared by the majority of stars analysed in NGC 1851 and by a small fraction of the present sample in M 80. However, most giants in M 80 have distinctly higher La abundances, and populate a region in the $[\mathrm{La} / \mathrm{Fe}] \mathrm{vs}$. $[\mathrm{Eu} / \mathrm{Fe}]$ plane where stars of the so-called $r+s$ component in M 22 are located (Roderer et al. 2011; they correspond to the $s$-rich group in Marino et al. 2011). This trend extends to higher metallicities thanks to a minority component in NGC 1851.

The spread observed in La for RGB stars in M 80 seems comparable to the range observed in the $r+s$ group in $\mathrm{M} 22$. On the other hand, although similarities between M 22 and M 80 include the total mass and metal abundance $([\mathrm{Fe} / \mathrm{H}] \sim-1.79 \mathrm{dex}$ in both), M 80 does not show a spread in $[\mathrm{Fe} / \mathrm{H}]$ as instead observed in M 22 (Da Costa et al. 2009; Marino et al. 2011).

In any case, if we plot the contribution from the $r+s$ group in M 22 from the analysis by Roederer et al. (2011) over the distribution of neutron-capture elements observed in M 80 (dotted line in the lower panel of Fig. 19, the agreement is not dramatically unsatisfactory.

We conclude that M 80 could be a less extreme case of a GC with a significant contribution from the $s$-process, and may be more similar to NGC 1851, although at lower metallicity. Although most of neutron-capture elements are only available for the limited sample of stars with UVES spectra, we found no statistically significant difference in the level of abundances of heavy elements between different stellar generations in $\mathrm{M} 80$. 


\section{Summary}

NGC $6093=$ M 80 is the last of the 24 GCs of our core program on the $\mathrm{Na}-\mathrm{O}$ anti-correlation in RGB stars and its relation with $\mathrm{HB}$ morphology and cluster parameters (see e.g. Carretta et al. 2006, 2009a,b). We present the analysis of this moderately massive, metal-poor, very dense GC, seen in the direction of the Galactic centre. We used FLAMES spectra of 82 red giant stars, confirmed members on the basis of their RV and metallicity and derived atmospheric parameters, metallicity, and chemical abundances (12 species for GIRAFFE spectra and more than 20 for UVES spectra).

The main goal is the determination of $\mathrm{O}$ and $\mathrm{Na}$ abundances, to study the shape and extension of the $\mathrm{Na}-\mathrm{O}$ anti-correlation, which is the main chemical signature of multiple populations in GCs (e.g. Carretta et al. 2010a). The ratios between first and second generation stars is normal, with fractions of $\mathrm{P}, \mathrm{I}$, and $\mathrm{E}$ stars at 36,56 , and $8 \%$, respectively. The three groups of stars appear to isolate naturally (see Fig. 8). The spread of both $\mathrm{O}$ and $\mathrm{Na}$ is large, about $1 \mathrm{dex}$, and the anti-correlation has a moderate extension (as measured by a IQR $[\mathrm{O} / \mathrm{Na}]=0.784$ ), which places NGC 6093 in the middle of the correlation with cluster mass (via $M_{V}$, see Fig. 11) and maximum temperature along the HB (see Fig. 16). The cluster also conforms to the relation between concentration and the residual around the best-fit IQR $[\mathrm{O} / \mathrm{Na}]$ and $M_{V}$.

Using the UVES spectra of 14 member stars, we can also study other p-capture elements. We find that $\mathrm{Al}$ is enhanced in I, E stars and is anti-correlated with $\mathrm{Mg}$, as usual. Briefly, this inner-halo cluster shows the classical chemical signatures of multiple stellar populations in the normal ways.

All $\alpha$, proton-capture, and iron peak elements behave in NGC 6093 like in a typical halo cluster. The neutron-capture elements (with the exception of $\mathrm{Ba}$ ) can be measured only in the UVES spectra. They conform to the run of field stars as function of metallicity (see Fig. 18). Comparing the values of [La/Fe] and $[\mathrm{Eu} / \mathrm{Fe}]$ with those of two clusters showing some spread in $r$-process or $s$-process elements (M 15 and M 22, respectively) we see that NGC 6093 may be a mild case of intrinsic dispersion is $s$-process elements (see Fig. 21), without showing, however, any indication of metallicity spread.

Acknowledgements. This publication makes use of data products from the Two Micron All Sky Survey, which is a joint project of the University of Massachusetts and the Infrared Processing and Analysis Center/California Institute of Technology, funded by the National Aeronautics and Space Administration and the National Science Foundation. This research has been funded by PRIN INAF 2011 "Multiple populations in globular clusters: their role in the Galaxy assembly" (PI E. Carretta), and PRIN MIUR 2010-2011, project "The Chemical and Dynamical Evolution of the Milky Way and Local Group Galaxies" (PI F. Matteucci). This research has made use of the SIMBAD database, operated at CDS, Strasbourg, France and of NASA's Astrophysical Data System.

\section{References}

Alonso, A., Arribas, S., \& Martinez-Roger, C. 1999, A\&AS, 140, 261 Alonso, A., Arribas, S., \& Martinez-Roger, C. 2001, A\&A, 376, 1039 Anderson, J., Sarajedini, A., Bedin, L., et al. 2008, AJ, 135, 2055 Arlandini, C., Käppeler, F., Wisshak, K., et al. 1999, ApJ, 525, 886 Bastian, N., Lamers, H. J. G. L. M., de Mink, S. E., et al. 2013, MNRAS, 436, 2398

Bellazzini, M., Bragaglia, A., Carretta, E., et al. 2012, A\&A, 538, A18 Bragaglia, A., Carretta, E., Gratton, R. G., et al. 2001, AJ, 121, 327 Bragaglia, A., Carretta, E., Gratton, R. G., et al. 2010, A\&A, 519, A60 Brocato, E., Castellani, V., Scotti, G. A., et al. 1998, A\&A, 335, 929 Carretta, E. 2006, AJ, 131, 1766

Carretta, E. 2014, ApJ, 795, L28
Carretta, E., Gratton R. G., Bragaglia, A., Bonifacio, P., \& Pasquini, L. 2004, A\&A, 416, 925

Carretta, E., Bragaglia, A., Gratton R. G., et al. 2006, A\&A, 450, 523

Carretta, E., Bragaglia, A., Gratton, R. G., et al. 2007a, A\&A, 464, 939

Carretta, E., Recio-Blanco, A., Gratton, R. G., Piotto, G., \& Bragaglia, A. 2007b, ApJ, 671, L125

Carretta, E., Bragaglia, A., Gratton, R. G., et al. 2009a, A\&A, 505, 117

Carretta, E., Bragaglia, A., Gratton, R. G., \& Lucatello, S. 2009b, A\&A, 505, 139

Carretta, E., Bragaglia, A., Gratton, R. G., D’Orazi, V., \& Lucatello, S. 2009c, A\&A, 508, 695

Carretta, E., Bragaglia, A., Gratton, R. G., et al. 2010a, A\&A, 516, A55

Carretta, E., Bragaglia, A., Gratton, R. G., et al. 2010b, A\&A, 520, A95

Carretta, E., Bragaglia, A., Gratton, R. G., D’Orazi, V., \& Lucatello, S. 2011a, A\&A, 535, A121

Carretta, E., Lucatello, S., Gratton, R. G., Bragaglia, A., \& D’Orazi, V. 2011b, A\&A, 533, A69

Carretta, E., Bragaglia, A., Gratton, R. G., Lucatello, S., \& D’Orazi, V. 2012, ApJ, 750, L14

Carretta, E., Bragaglia, A., Gratton, R. G., et al. 2013, A\&A, 557, A138

Carretta, E., Bragaglia, A., Gratton, R. G., et al. 2014a, A\&A, 561, A87

Carretta, E., Bragaglia, A., Gratton, R. G., et al. 2014b, A\&A, 564, A60

Carretta, E., D’Orazi, V., Gratton, R. G., \& Lucatello, S. 2014c, A\&A, 563, A32

Cassisi, S., Mucciarelli, A., Pietrinferni, A., Salaris, M., \& Ferguson, J., 2013, A\&A, 554, A19

Cavallo, R. M., Suntzeff, N. B., \& Pilachowski, C. A. 2004, AJ, 127, 3411

Cordero, M. J., Pilachowski, C. A., Johnson C. I., et al. 2014, ApJ, 780, 94

Da Costa, G. S., Held, E. V., Saviane, I., \& Gullieuszik, M. 2009, ApJ, 705, 1481

Decressin, T., Meynet, G., Charbonnel C. Prantzos, N., \& Ekstrom, S. 2007, A\&A, 464, 1029

de Mink, S. E., Pols, O. R., Langer, N., \& Izzard, R. G. 2009, A\&A, 507, L1

D'Ercole, A., D’Antona, F., Carini, R., Vesperini, E., \& Ventura, P. 2012, MNRAS, 423, 1521

Denisenkov, P. A., \& Denisenkova, S. N. 1989, A. Tsir., 1538, 11

Dinescu, D. I., van Altena, W. F., Girard, T. M., \& López, C. E. 1999, AJ, 117, 277

Dobrovolskas, V., Kučinskas, A., Bonifacio, P., et al. 2014, A\&A, 565, A121

D'Orazi, V., \& Marino, A. F. 2010, ApJ, 716, L166

D'Orazi, V., Gratton, R. G., Lucatello, S., et al. 2010a, ApJ, 719, L213

D'Orazi, V., Lucatello, S., Gratton, R. G., et al. 2010b, ApJ, 713, L1

D’Orazi, V., Biazzo, K., Desidera, S., et al. 2012, MNRAS, 423, 2789

D'Orazi, V., Campbell, S. W., Lugaro, M., et al. 2013, MNRAS, 433, 366

D'Orazi, V., Angelou, G. C., Gratton, R. G., et al. 2014, ApJ, 791, 39

Feltzing, S., \& Gustafsson, B. 1998, A\&AS, 129, 237

Ferraro, F. R., Paltrinieri, B., Fusi Pecci, F., Rood, R. T., \& Dorman, B. 1998, ApJ, 500, 311

Ferraro, F. R., Paltrinieri, B., Rood, R. T., \& Dorman, B. 1999, ApJ, 522, 983

González Hernández, J. I., Bonifacio, P., Caffau, E., et al. 2009, A\&A, 505, 13

Gratton, R. G. 1988, Rome Obs. Preprint Ser., 29

Gratton, R. G., Carretta, E., Eriksson, K., \& Gustafsson, B. 1999, A\&A, 350, 955

Gratton, R. G., Bonifacio, P., Bragaglia, A., et al. 2001, A\&A, 369, 87

Gratton, R. G., Carretta, E., Claudi, R., Lucatello, S., \& Barbieri, M. 2003, A\&A 404, 187

Gratton, R. G., Sneden, C., \& Carretta, E. 2004, ARA\&A, 42, 385

Gratton, R. G., Lucatello, S., Bragaglia, A., et al. 2006, A\&A, 455, 271

Gratton, R. G., Lucatello, S., Bragaglia, A., et al. 2007, A\&A, 464, 953

Gratton, R. G., Carretta, E., Bragaglia, A., Lucatello, S., \& D’Orazi, V. 2010, A\&A, 517, A81

Gratton, R. G., Lucatello, S., Carretta, E., et al. 2011, A\&A, 534, A123

Gratton, R. G., Villanova, S., Lucatello, S., et al. 2012a, A\&A, 544, A12

Gratton, R. G., Carretta, E., \& Bragaglia, A. 2012b, A\&ARv, 20, 50

Gratton, R. G., Lucatello, S., Sollima, A., et al. 2014, A\&A, 563, A13

Grundahl, F., VandenBerg, D. A., \& Andersen, M. I. 1998, ApJ, 500, L179

Gruyters, P., Nordlander, T., \& Korn, A. J. 2014, A\&A, 567, A72

Gunn, J. E., \& Griffin, R. F. 1979, AJ, 84, 752

Han, S.-I., Lee, Y.-W., Joo, S.-J., et al. 2009, ApJ, 707, L190

Harris, W. E. 1996, AJ, 112, 1487

Heinke, C. O., Grindlay, J. E., Edmonds, P. D., et al. 2003, ApJ, 598, 516

King, I. R. 1966, AJ, 71, 64

Koch, A., \& McWilliam, A. 2014, A\&A, 565, A23

Kopacki, G. 2013, AcA, 63, 91

Kraft, R. P. 1994, PASP, 106, 553

Küpper, A. H. W., Kroupa, P., Baumgardt, H., \& Heggie, D. C. 2010, MNRAS, 407,2241

Kurucz, R. L. 1993, CD-ROM 13, Smithsonian Astrophysical Observatory, Cambridge

Langer, G. E., Hoffman, R., \& Sneden, C. 1993, PASP, 105, 301 
Lee, Y.-W. 1990, ApJ, 363, 159

Lee, J.-W., Lee, J., Kang, Y.-W., et al. 2009, ApJ, 695, L78

Lind, K., Korn, A. J., Barklem, P. S., \& Grundahl, F. 2008, A\&A, 490, 777

Lind, K., Primas, F., Charbonnel, C., Grundahl, F., \& Asplund, M. 2009, A\&A, 503,545

Lovisi, L., Mucciarelli, A., Lanzoni, B., et al. 2012, ApJ, 754, 91

Mackey, A. D., \& van den Bergh, S. 2005, MNRAS, 360, 631

Magain, P. 1984, A\&A, 134, 189

Marino, A. F., Villanova, S., Piotto, G., et al. 2008, A\&A, 490, 625

Marino, A. F., Sneden, C., Kraft, R. P., et al. 2011, A\&A, 532, A8

Marino, A. F., Milone, A. P., Sneden, C., et al. 2012, A\&A, 541, A15

Marino, A. F., Milone, A. P., \& Lind, K. 2013, ApJ, 768, 27

McWilliam, A., Preston, G. W. Sneden, C., \& Searle, L. 1995, AJ, 109, 2757

Milone, A., Piotto, G., Bedin, L., et al. 2012, ApJ, 744, 58

Milone, A. P., Marino, A. F., Piotto, G., et al. 2013, ApJ, 767, 120

Mishenina, T. V., Kovtyukh, V. V., Soubiran, C., Travaglio, C., \& Busso, M. 2002, A\&A, 396, 189

Momany, Y., Bedin, L. R., Cassisi, S., et al. 2004, A\&A, 420, 605

Monelli, M., Milone, A. P., Stetson, P. B., et al. 2013, MNRAS, 431, 2126

Nardiello, D., Milone, A. P., Piotto, G., et al. 2015, A\&A, 573, A70

Nataf, D. M., Gould, A., Pinsonneault, M. H., \& Stetson, P. B. 2011, ApJ, 736, 94

Noyola, E., \& Gebhardt, K. 2006, AJ, 132, 447

Otsuki, K., Honda, S., Aoki, W., Kajino, T., \& Mathews, G. J. 2006, ApJ, 641, L117

Paust, N. E. Q., Reid, I. N., Piotto, G., et al. 2010, AJ, 139, 476

Pryor, C., \& Meylan, G. 1993, ASPC, 50, 357

Ramírez, S., \& Cohen, J. G. 2002, AJ, 123, 3277

Ramírez, S., \& Cohen, J. G. 2003, AJ, 125, 224
Recio-Blanco, A., Aparicio, A., Piotto, G., De Angeli, F., \& Djorgovski, S. G. 2006, A\&A, 452, 875

Reddy, B. E., Tomkin, J., Lambert, D. L., \& Allende Prieto, C. 2003, MNRAS, 340,304

Roederer, I. U. 2011, ApJ, 732, L17

Roederer, I. U., Cowan, J. J., Karakas, A. I., et al. 2010, ApJ, 724, 975

Roederer, I. U., Marino, A. F., \& Sneden, C. 2011, ApJ, 742, 37

Ryan, S. G., Norris, J. E., \& Beers, T. C. 1996, ApJ, 471, 254

Salaris, M., Weiss, A., Ferguson, J. W., \& Fusilier, D. J. 2006, ApJ, 645, 1131

Sarajedini, A., Bedin, L., Chaboyer, B., et al. 2007, AJ, 133, 1658

Sbordone, L., Salaris, M., Weiss, A., \& Cassisi, S. 2011, A\&A, 534, A9

Shen, Z.-X., Bonifacio, P., Pasquini, L., \& Zaggia, S. 2010, A\&A, 524, A2

Simmerer, J., Sneden, C., Cowan, J. J., et al. 2004, ApJ, 617, 1091

Skrutskie, M. F., Cutri, R. M., Stiening, R., et al. 2006, AJ, 131, 1163

Smith, G. H. 1987, PASP, 99, 67

Sneden, C. 2000, in The Galactic Halo: From Globular Cluster to Field Stars, Proc. 35th Liege Int. Astrophys. Colloq., 1999. eds. A. Noels, P. Magain, D. Caro, et al., Liege, Belgium, 159

Sneden, C., Cowan, J. J., Lawler, J. E., et al. 2003, ApJ, 591, 936

Sobeck, J. S., Kraft, R. P., Sneden, C., et al. 2011, AJ, 141, 175

Sollima, A., Bellazzini, M., \& Lee, J.-W. 2012, ApJ, 755, 156

Venn, K.A, Irwin, M., Shetrone, M. D., et al. 2004, AJ, 128, 1177

Ventura, P. D'Antona, F., Mazzitelli, I., \& Gratton, R. 2001, ApJ, 550, L65

Worley, C. C., Hill, V., Sobeck, J., \& Carretta, E. 2013, A\&A, 553, A47

Yong, D., Grundahl, F., Nissen, P. E., Jensen, H. R., \& Lambert, D. L. 2005, A\&A, 438, 875

Yong, D., Mélendez, J., Grundahl, F., et al. 2013, MNRAS, 434, 3452

Zoccali, M., Cassisi, S., Piotto, G., Bono, G., \& Salaris, M. 1999, ApJ, 518, L49 


\section{Appendix A: Error estimates}

We refer the reader to the analogous Appendices in Carretta et al. (2009a,b) for a detailed discussion of the procedure adopted for error estimates.

Table A.1. Sensitivities of abundance ratios to variations in the atmospheric parameters, and errors in the equivalent widths, and errors in abundances for stars of NGC 6093 observed with UVES.

\begin{tabular}{|c|c|c|c|c|c|c|c|c|}
\hline Element & $\begin{array}{c}\text { Average } \\
n . \text { lines }\end{array}$ & $\begin{array}{l}T_{\text {eff }} \\
(\mathrm{K})\end{array}$ & $\begin{array}{l}\log g \\
(\operatorname{dex})\end{array}$ & $\begin{array}{r}{[\mathrm{A} / \mathrm{H}]} \\
(\mathrm{dex})\end{array}$ & $\begin{array}{r}v_{\mathrm{t}} \\
\mathrm{km} \mathrm{s}^{-1}\end{array}$ & $\begin{array}{l}E W s \\
(\operatorname{dex})\end{array}$ & $\begin{array}{r}\text { Total } \\
\text { internal }\end{array}$ & $\begin{array}{r}\text { Total } \\
\text { systematic }\end{array}$ \\
\hline Variation & & 50 & 0.20 & 0.10 & 0.10 & & & \\
\hline internal & & 4 & 0.04 & 0.02 & 0.10 & 0.01 & & \\
\hline systematic & & 58 & 0.06 & 0.08 & 0.03 & & & \\
\hline$[\mathrm{Fe} / \mathrm{H}] \mathrm{I}$ & 56 & +0.065 & -0.005 & -0.013 & -0.018 & 0.011 & 0.022 & 0.076 \\
\hline$[\mathrm{Fe} / \mathrm{H}] \mathrm{II}$ & 8 & -0.033 & +0.085 & +0.023 & -0.007 & 0.029 & 0.035 & 0.046 \\
\hline$[\mathrm{O} / \mathrm{Fe}] \mathrm{I}$ & 2 & -0.048 & +0.083 & +0.041 & +0.015 & 0.057 & 0.062 & 0.088 \\
\hline$[\mathrm{Na} / \mathrm{Fe}] \mathrm{I}$ & 3 & -0.022 & -0.047 & -0.020 & +0.012 & 0.047 & 0.049 & 0.066 \\
\hline$[\mathrm{Mg} / \mathrm{Fe}] \mathrm{I}$ & 2 & -0.020 & -0.011 & -0.002 & +0.008 & 0.057 & 0.058 & 0.035 \\
\hline$[\mathrm{Al} / \mathrm{Fe}] \mathrm{I}$ & 2 & -0.019 & -0.010 & -0.001 & +0.016 & 0.057 & 0.060 & 0.194 \\
\hline$[\mathrm{Si} / \mathrm{Fe}] \mathrm{I}$ & 7 & -0.056 & +0.023 & +0.014 & +0.014 & 0.031 & 0.034 & 0.066 \\
\hline$[\mathrm{Ca} / \mathrm{Fe}] \mathrm{I}$ & 16 & +0.008 & -0.020 & -0.009 & -0.009 & 0.020 & 0.023 & 0.012 \\
\hline$[\mathrm{Sc} / \mathrm{Fe}] \mathrm{II}$ & 8 & +0.030 & -0.016 & +0.001 & -0.008 & 0.029 & 0.030 & 0.036 \\
\hline$[\mathrm{Ti} / \mathrm{Fe}] \mathrm{I}$ & 9 & +0.029 & -0.015 & -0.008 & +0.008 & 0.027 & 0.028 & 0.036 \\
\hline$[\mathrm{Ti} / \mathrm{Fe}] \mathrm{II}$ & 9 & +0.024 & -0.024 & -0.007 & -0.011 & 0.027 & 0.030 & 0.029 \\
\hline [V/Fe]I & 8 & +0.033 & -0.014 & -0.009 & +0.004 & 0.029 & 0.029 & 0.039 \\
\hline$[\mathrm{Cr} / \mathrm{Fe}] \mathrm{I}$ & 12 & +0.048 & -0.021 & -0.014 & -0.023 & 0.023 & 0.033 & 0.057 \\
\hline$[\mathrm{Cr} / \mathrm{Fe}] \mathrm{II}$ & 7 & +0.002 & -0.019 & -0.014 & +0.001 & 0.031 & 0.031 & 0.009 \\
\hline$[\mathrm{Mn} / \mathrm{Fe}] \mathrm{I}$ & 3 & +0.005 & -0.011 & -0.003 & +0.009 & 0.047 & 0.048 & 0.009 \\
\hline$[\mathrm{Co} / \mathrm{Fe}] \mathrm{I}$ & 3 & -0.010 & -0.004 & +0.003 & +0.015 & 0.047 & 0.049 & 0.014 \\
\hline$[\mathrm{Ni} / \mathrm{Fe}] \mathrm{I}$ & 17 & -0.004 & +0.010 & +0.008 & +0.005 & 0.020 & 0.020 & 0.008 \\
\hline$[\mathrm{Cu} / \mathrm{Fe}] \mathrm{I}$ & 2 & -0.001 & +0.001 & +0.001 & -0.001 & 0.057 & 0.057 & 0.011 \\
\hline$[\mathrm{Zn} / \mathrm{Fe}] \mathrm{I}$ & 1 & -0.087 & +0.039 & +0.019 & -0.005 & 0.081 & 0.082 & 0.103 \\
\hline [Y/Fe]II & 12 & +0.036 & -0.023 & -0.004 & -0.019 & 0.023 & 0.031 & 0.044 \\
\hline$[\mathrm{Zr} / \mathrm{Fe}] \mathrm{I}$ & 3 & +0.069 & -0.010 & -0.012 & +0.016 & 0.047 & 0.050 & 0.082 \\
\hline$[\mathrm{Zr} / \mathrm{Fe}] \mathrm{II}$ & 1 & +0.030 & -0.016 & -0.002 & +0.003 & 0.081 & 0.081 & 0.039 \\
\hline$[\mathrm{Ba} / \mathrm{Fe}] \mathrm{II}$ & 3 & +0.052 & -0.026 & +0.004 & -0.079 & 0.047 & 0.092 & 0.078 \\
\hline$[\mathrm{La} / \mathrm{Fe}] \mathrm{II}$ & 1 & +0.058 & +0.008 & +0.002 & -0.010 & 0.081 & 0.082 & 0.073 \\
\hline$[\mathrm{Ce} / \mathrm{Fe}] \mathrm{II}$ & 1 & +0.042 & -0.018 & -0.001 & -0.001 & 0.081 & 0.081 & 0.063 \\
\hline$[\mathrm{Pr} / \mathrm{Fe}] \mathrm{II}$ & 4 & +0.045 & -0.016 & +0.000 & +0.002 & 0.041 & 0.041 & 0.058 \\
\hline$[\mathrm{Nd} / \mathrm{Fe}] \mathrm{II}$ & 7 & +0.049 & -0.019 & -0.001 & -0.014 & 0.031 & 0.034 & 0.061 \\
\hline$[\mathrm{Sm} / \mathrm{Fe}] \mathrm{II}$ & 1 & +0.052 & -0.021 & -0.001 & -0.003 & 0.081 & 0.081 & 0.065 \\
\hline$[\mathrm{Eu} / \mathrm{Fe}] \mathrm{II}$ & 2 & +0.033 & -0.008 & +0.004 & +0.002 & 0.047 & 0.057 & 0.039 \\
\hline
\end{tabular}

Table A.2. Sensitivities of abundance ratios to variations in the atmospheric parameters, errors in the equivalent widths, and errors in abundances for stars of NGC 6093 observed with GIRAFFE.

\begin{tabular}{lrrrrrrrr}
\hline \hline Element & $\begin{array}{r}\text { Average } \\
n . \text { lines }\end{array}$ & $\begin{array}{r}T_{\text {eff }} \\
(\mathrm{K})\end{array}$ & $\begin{array}{r}\log g \\
(\mathrm{dex})\end{array}$ & $\begin{array}{r}{[\mathrm{A} / \mathrm{H}]} \\
(\mathrm{dex})\end{array}$ & $\begin{array}{r}v_{\mathrm{t}} \\
\mathrm{km} \mathrm{s}^{-1}\end{array}$ & $\begin{array}{r}E W \mathrm{~s} \\
(\mathrm{dex})\end{array}$ & $\begin{array}{r}\text { Total } \\
\text { internal }\end{array}$ & $\begin{array}{r}\text { Total } \\
\text { systematic }\end{array}$ \\
\hline Variation & & 50 & 0.20 & 0.10 & 0.10 & & & \\
internal & & 4 & 0.04 & 0.02 & 0.36 & 0.02 & & \\
systematic & & 58 & 0.06 & 0.07 & 0.04 & & & \\
\hline$[\mathrm{Fe} / \mathrm{H}] \mathrm{I}$ & 19 & +0.060 & -0.009 & -0.011 & -0.017 & 0.022 & 0.065 & 0.070 \\
{$[\mathrm{Fe} / \mathrm{H}] \mathrm{II}$} & 2 & -0.019 & +0.079 & +0.013 & -0.005 & 0.067 & 0.071 & 0.033 \\
{$[\mathrm{O} / \mathrm{Fe}] \mathrm{I}$} & 1 & -0.043 & +0.085 & +0.032 & +0.020 & 0.095 & 0.121 & 0.063 \\
{$[\mathrm{Na} / \mathrm{Fe}] \mathrm{I}$} & 2 & -0.026 & -0.028 & -0.006 & +0.013 & 0.067 & 0.082 & 0.050 \\
{$[\mathrm{Mg} / \mathrm{Fe}] \mathrm{I}$} & 1 & -0.025 & -0.001 & +0.001 & +0.012 & 0.095 & 0.104 & 0.030 \\
{$[\mathrm{Si} / \mathrm{Fe}] \mathrm{I}$} & 3 & -0.044 & +0.021 & +0.008 & +0.015 & 0.055 & 0.077 & 0.052 \\
{$[\mathrm{Ca} / \mathrm{Fe}] \mathrm{I}$} & 4 & -0.010 & -0.009 & -0.002 & -0.002 & 0.048 & 0.048 & 0.012 \\
{$[\mathrm{Sc} / \mathrm{Fe}] \mathrm{II}$} & 4 & -0.052 & +0.082 & +0.027 & +0.009 & 0.048 & 0.060 & 0.065 \\
{$[\mathrm{Ti} / \mathrm{Fe}] \mathrm{I}$} & 3 & +0.009 & -0.004 & -0.002 & +0.013 & 0.055 & 0.072 & 0.012 \\
{$[\mathrm{~V} / \mathrm{Fe}] \mathrm{I}$} & 4 & +0.026 & -0.007 & -0.003 & +0.016 & 0.048 & 0.075 & 0.031 \\
{$[\mathrm{Cr} / \mathrm{Fe}] \mathrm{I}$} & 2 & +0.006 & -0.006 & -0.001 & +0.019 & 0.067 & 0.096 & 0.013 \\
{$[\mathrm{Ni} / \mathrm{Fe}] \mathrm{I}$} & 4 & -0.001 & +0.009 & +0.004 & +0.012 & 0.048 & 0.064 & 0.006 \\
{$[\mathrm{Ba} / \mathrm{Fe}] \mathrm{II}$} & 1 & -0.038 & +0.076 & +0.026 & -0.060 & 0.095 & 0.237 & 0.067 \\
\hline
\end{tabular}

